\title{
EXPERIMENTAL PNEUMOCOCCUS LOBAR PNEUMONIA IN THE DOG
}

\author{
II. Pathology \\ By OSWALD H. ROBERTSON, LOWELL T. COGGESHALL AND \\ EDWARD E. TERRELL
}

(From the Department of Medicine, University of Chicago, Chicago)

(Received for publication October 31, 1932)

In a previous paper (1) we described a method for the production of experimental lobar pneumonia in the dog with relatively small doses of pneumococcus Types I and II. The course of the experimental disease resembled in many respects lobar pneumonia as observed in man. Further analogy with the human disease is revealed by the study of its pathology. The present communication consists of a description of gross and microscopic changes found in the lungs of dogs dying spontaneously of the disease as well as of those killed at various stages in its course.

\section{MATERIAL AND METHODS}

Autopsies were performed in 58 instances of pneumococcus pneumonia produced experimentally in dogs; 30 of these dogs died spontaneously or were killed while very ill and 28 were sacrificed at intervals of one hour to eight days from the beginning of the disease. Table I, the basis of this study. includes only those dogs dying spontaneously or killed at forty-eight hours and later. In addition, 6 dogs were killed following sterile starch injection. Two methods were employed for killing the dog without producing lung injury, electrocution and lethal doses of nembutal. The former was used for the most part and was entirely satisfactory when the contacts were good-mucous membrane and shaved skin. A 110 volt alternating current was employed. In the early part of the work before the importance of good contact was realized, petechial hemorrhages were observed in one or more lobes of the lungs in those animals in which death was not instantaneous. Nembutal in amounts of 400 to $500 \mathrm{mgm}$. injected rapidly into a vein produced an almost instantaneous and quiet death without any detectable disturbance of the lungs. Examinations were made in most cases immediately after death. The trachea was clamped before opening the thorax in order to estimate the relative sizes of the involved and normal lungs. In many instances $x$-rays of the excised lungs were taken.

Tissues were fixed in Zenker's fluid with 5 per cent acetic acid added, and mounted in paraffin. In addition to hematoxylin and eosin, sections were stained with a modification of the Gram-Weigert method devised by H. M. Wallace (2), which made it possible to study the relationships of cell structure, pneumococci and fibrin in the same preparation. The method is as follows: 
After a very light hematoxylin stain the sections are thoroughly washed in tap water, then dipped into a one-half per cent aqueous eosin (Grubler's wasserlich) for half a minute and washed quickly again. (Washing is done in large volumes of water.) They are then stained in Weigert's aniline methyl violet made up as follows:

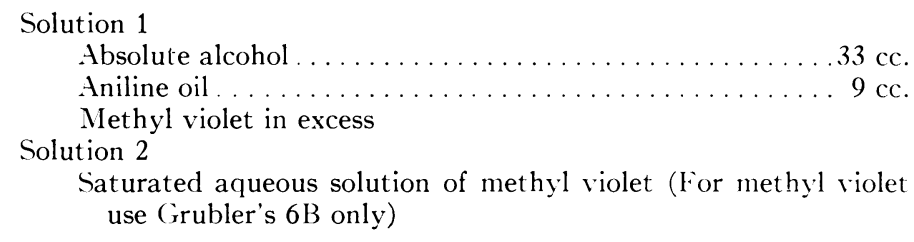

One part of Solution 1 is used to nine parts of Solution 2 for the stain. The two solutions will keep separately, but after mixing, the stain will last only about ten days. It seems to give the best result about three to eight days after it is made.

The tissues are stained in the methrl violet about two hours, washed well in tap water and put into Lugol's solution for ten or fifteen minutes, after which they are again washed. Each slide is then blotted thoroughly with filter-paper and subsequently differentiated in a mixture of one part aniline oil to two parts xylol. After washing in several changes of xylol they are mounted in balsam.

The fibrin and gram-positive organisms are stained a deep purple; the dark blue nuclei are well differentiated. All outlines of cells can be seen distinctly, due to the counterstain. Blood corpuscles are generally of a pale blue color, though at times they remain pink.

\section{Pathology of pneumococcus pneumonia in dogs}

It was pointed out in the preceding paper that the course of the experimental disease depended, within certain limits, on the size of the infecting dose. In the range of dosage from which the majority of the dogs recovered (less than $0.1 \mathrm{cc}$. of culture) the pneumonic process tended to remain localized in the lungs, and intense consolidation of the affected lobes resulted. With amounts of pneumococcus culture usually producing a lethal outcome the initial lung lesion was of ten localized for the first two or three days of the disease, but for some reason the consolidation failed to progress and the infection became a general one. Such animals showed changes quite different from those in which the infection remained primarily localized in the lungs. A third form of the disease occurred in certain animals, showing an unusual degree of temperature depression from the initial morphine injection, or in those receiving very large infecting doses. This was marked by rapid spread of the pulmonary lesion, bacteremia and death within two to three days. These several conditions will be dealt with separately. The details of dosage, time of death, extent and character of lung involvement, bacteriology, etc., are given in Table I. Table II summarizes the different types of lesion found at autopsy. 
O. H. ROBERTSON, L. T. COGGESHALL AND E. E. TERRELL 435

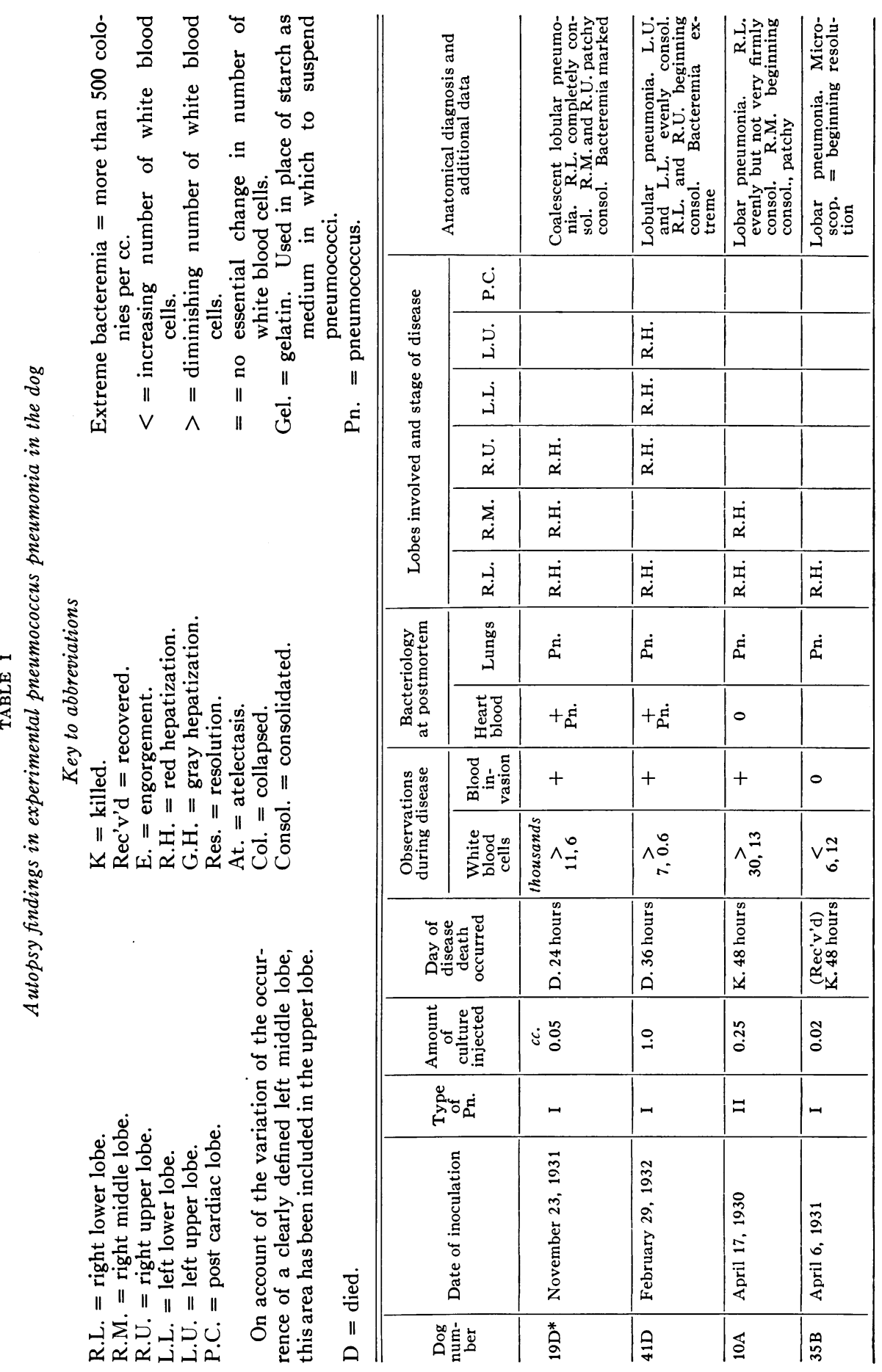




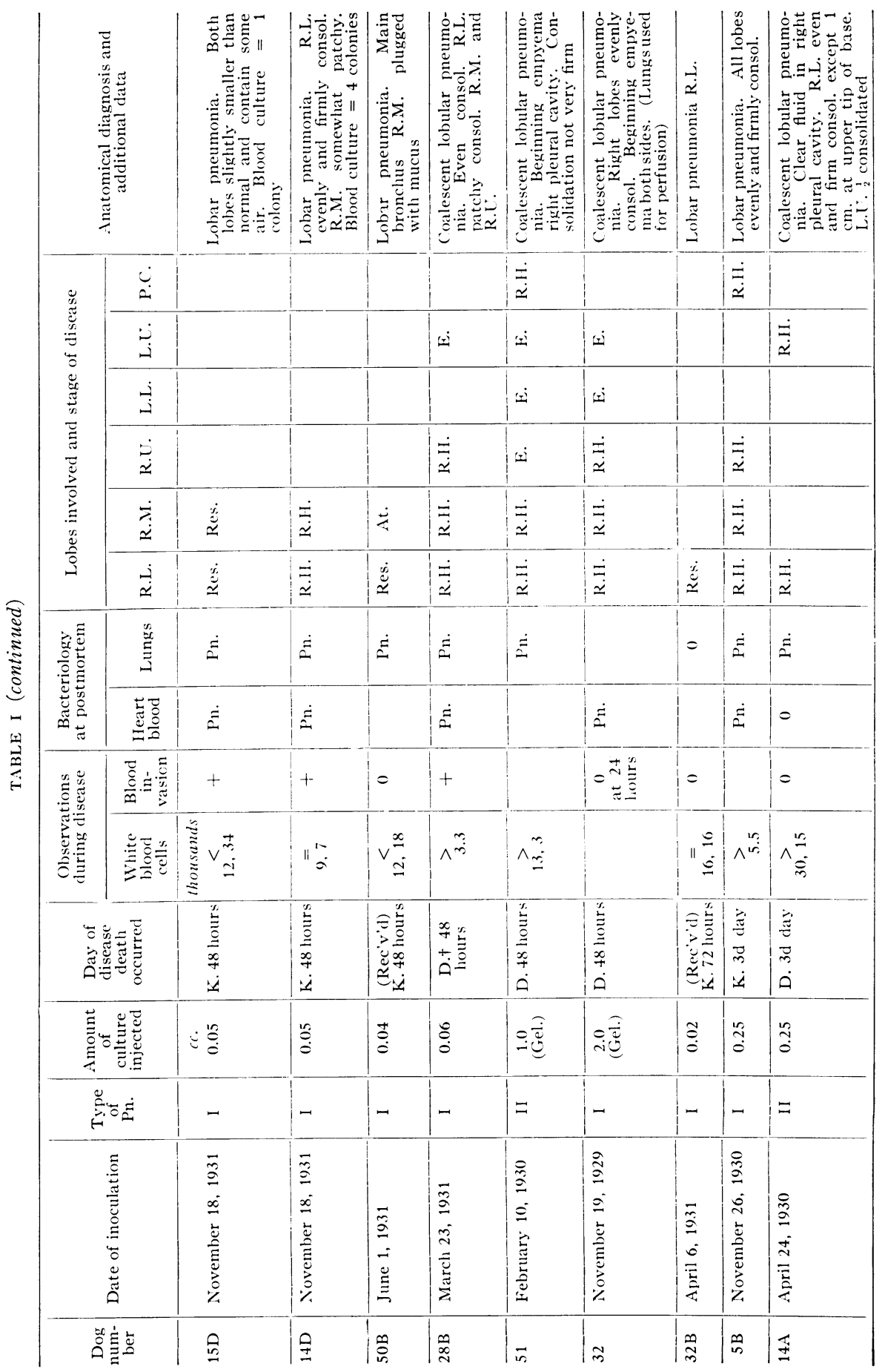


o. H. ROBERTSON, L. T. COGGESHALL AND E. E. TERRELL 437

\begin{tabular}{|c|c|c|c|c|c|c|c|c|c|c|}
\hline & 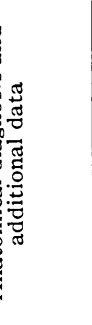 & 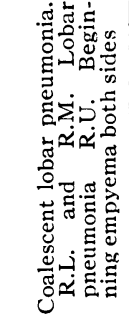 & 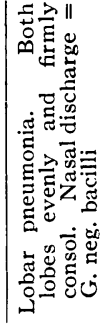 & 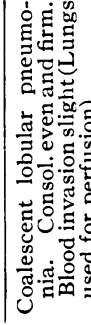 & 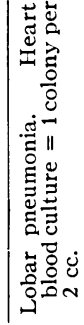 & 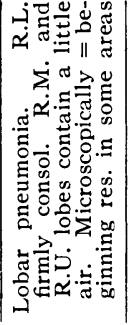 & 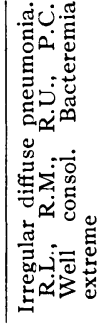 & 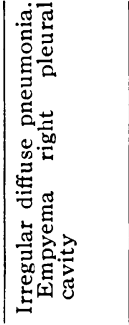 & 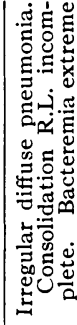 & 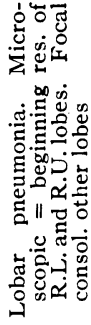 \\
\hline 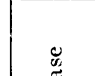 & ن. & & & $\mid \dot{a}$ & $\mid \begin{array}{l}\dot{\infty} \\
\ddot{\leftrightarrow}\end{array}$ & & 离 & & wi & $\stackrel{\dot{\pi}}{\varkappa}$ \\
\hline 离 & نُ & & & & & $\mid \ddot{\ddot{~}}$ & & $\ddot{\ddot{\mu}}$ & जi & \\
\hline 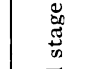 & ذذذ & & & & & & & $\ddot{\dot{a}}$ & |ن & \\
\hline 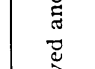 & نُ & تُ & & $\mid \ddot{\ddot{\alpha}}$ & & $\mid \ddot{\square}$ & $\mid$ & 过 & ند & 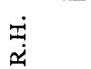 \\
\hline 恶 & $\dot{\Sigma}_{\alpha}$ & 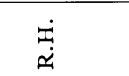 & $\ddot{\ddot{z}}$ & 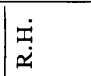 & $\mid \begin{array}{l}\dot{0} \\
\stackrel{\leftrightarrow}{\mathscr{\Delta}}\end{array}$ & 䓛 & $\underset{\dot{a}}{\dot{\alpha}}$ & 蚛 & ن & 虫 \\
\hline$\stackrel{5}{9}$ & $\dot{\dot{\alpha}}$ & 菂 & ت & 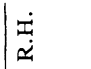 & $\mid \begin{array}{l}\dot{\mathscr{g}} \\
\stackrel{\mathscr{\varkappa}}{2}\end{array}$ & $\underset{\check{~}}{\ddot{2}}$ & | & 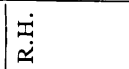 & 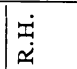 & यं \\
\hline 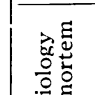 & 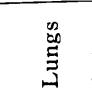 & 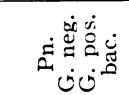 & 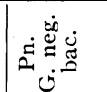 & & $\dot{E}$ & $\dot{2}$ & $\dot{E}$ & 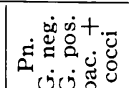 & $\dot{m}$ & घ \\
\hline 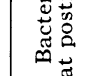 & 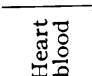 & & 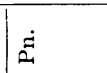 & $\dot{\Xi}$ & 豆 & 0 & $\dot{\mid g}$ & & $\dot{\Xi}$ & $\dot{\Delta}$ \\
\hline 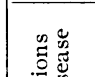 & 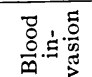 & 0 & & + & + & 0 & + & $1+$ & + & + \\
\hline 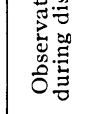 & 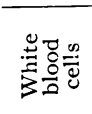 & 离 & $\Lambda_{0}^{m}$ & & $v_{\infty}=$ & $v_{\stackrel{i}{\pi}}^{\pi}$ & $\Lambda_{0}^{*}$ & 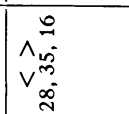 & $\wedge_{\infty}^{\infty}$ & 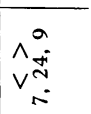 \\
\hline$\vec{A}$ & & $\begin{array}{l}\overrightarrow{\vec{d}} \\
\vec{j} \\
\vec{j} \\
\dot{\theta}\end{array}$ & 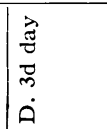 & \begin{tabular}{|l|}
$\vec{z}$ \\
$\vec{z}$ \\
$\vec{D}$ \\
$\dot{m}$ \\
$\dot{a}$
\end{tabular} & 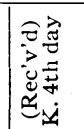 & 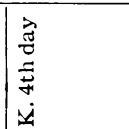 & 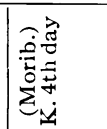 & 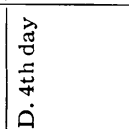 & 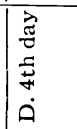 & 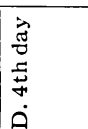 \\
\hline & 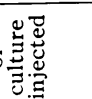 & ن & 它 & 串 & 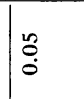 & $\mid \begin{array}{l}8 \\
0 \\
0\end{array}$ & 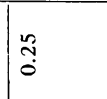 & 苛 & $\stackrel{n}{0}$ & $\overline{0}$ \\
\hline & 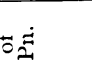 & $=$ & $=$ & - & - & - & - & - & - & - \\
\hline & 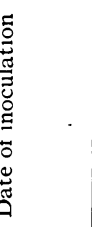 & 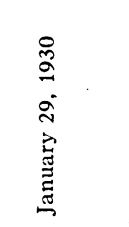 & 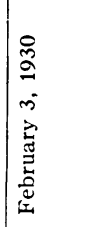 & 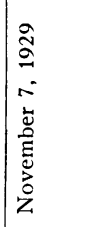 & 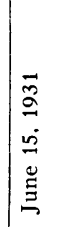 & 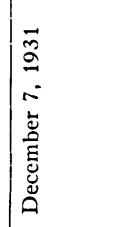 & 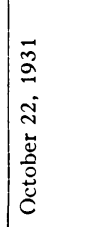 & 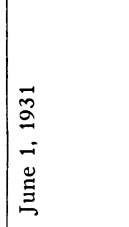 & 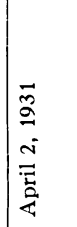 & 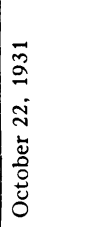 \\
\hline$\stackrel{80}{\circ}$ & 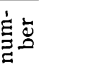 & $q$ & 18 & | & $\left.\right|_{N} ^{u}$ & 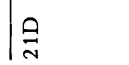 & | & $\mid \begin{array}{l}\infty \\
z\end{array}$ & 离 & | \\
\hline
\end{tabular}




\begin{tabular}{|c|c|c|c|c|c|c|c|c|c|}
\hline & & 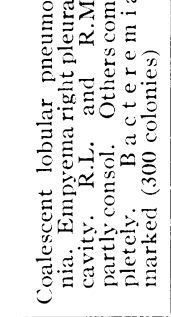 & 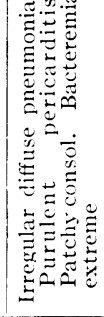 & 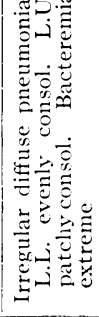 & 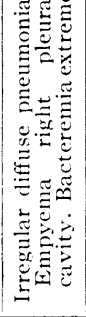 & 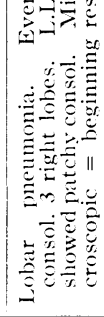 & 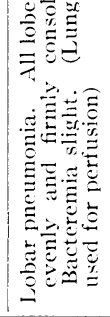 & 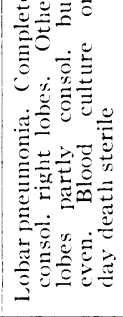 & 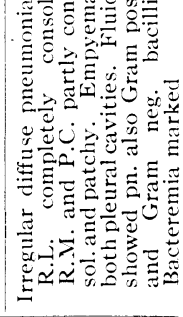 \\
\hline$\tilde{u}$ & $\dot{~}$ & & & & & & $\stackrel{\check{\check{\simeq}}}{\doteq}$ & 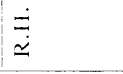 & 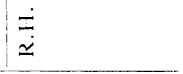 \\
\hline 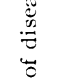 & تُ & $\dot{\square}$ & & $\dot{\overrightarrow{\underline{x}}}$ & & & & $\dot{\overrightarrow{\ddot{x}}}$ & \\
\hline 苞 & 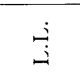 & & & $\dot{\overrightarrow{\underline{u}}}$ & & $\dot{\vec{x}}$ & 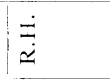 & $\begin{array}{l}\overline{\bar{x}} \\
\underline{u}\end{array}$ & \\
\hline $\begin{array}{l}\stackrel{\Xi}{\Xi} \\
\stackrel{\Xi}{\square}\end{array}$ & $\stackrel{\vec{\sim}}{\check{\sim}}$ & $\dot{\bar{\Xi}}$ & & & & $\overrightarrow{\bar{\approx}}$ & $\ddot{\overline{\underline{u}}}$ & 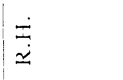 & \\
\hline 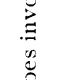 & $\begin{array}{l}\dot{\bar{Z}} \\
\check{\beth}\end{array}$ & $\dot{\overrightarrow{\check{x}}}$ & & $\frac{\dot{n}}{\ddot{n}}$ & & $\stackrel{\vec{u}}{\underline{\underline{u}}}$ & $\stackrel{\overrightarrow{\underline{\sim}}}{\ddot{\sim}}$ & $\dot{\overline{\ddot{x}}}$ & $\begin{array}{l}\ddot{\square} \\
\ddot{\beth}\end{array}$ \\
\hline$\overline{3}$ & $\stackrel{\dot{\square}}{\check{\check{L}}}$ & $\underset{\check{Z}}{\ddot{Z}}$ & $\dot{\overrightarrow{\underline{a}}}$ & 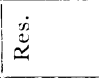 & 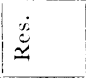 & $\dot{\overrightarrow{\underline{x}}}$ & $\underset{\check{~}}{\check{\beth}}$ & $\begin{array}{l}\doteq \\
\approx\end{array}$ & $\begin{array}{l}\vec{\beth} \\
\ddot{\beth}\end{array}$ \\
\hline 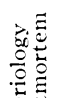 & 先 & $\dot{\Xi}$ & & $\dot{\equiv}$ & $\doteq$ & $\doteq$ & & & $\dot{\Xi}$ \\
\hline 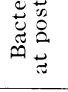 & 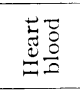 & $\dot{\equiv}$ & $\doteq$ & $\dot{\Xi}$ & $1 \doteq$ & $=$ & & & \\
\hline 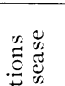 & 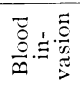 & + & + & + & + & $=$ & + & 0 & + \\
\hline 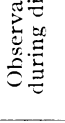 & 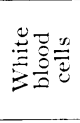 & 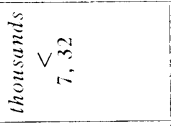 & $\wedge_{i=}^{+}$ & 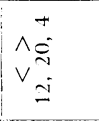 & in & $\begin{array}{c}v^{*}= \\
=\end{array}$ & $\wedge_{i}^{+}$ & & $\wedge^{m}$ \\
\hline & & 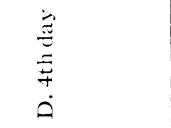 & 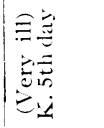 & 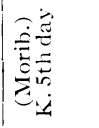 & 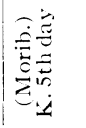 & 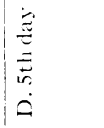 & 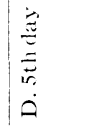 & 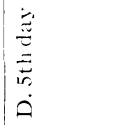 & 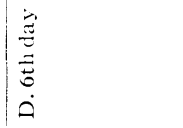 \\
\hline & & 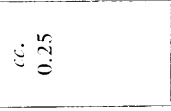 & $\stackrel{ \pm}{\Xi}$ & $\stackrel{\stackrel{\mathscr{c}}{0}}{\mathrm{E}}$ & $\vec{\Xi}$ & 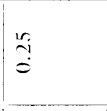 & 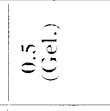 & 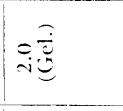 & $\overrightarrow{0}$ \\
\hline & & - & - & - & - & $=$ & - & - & - \\
\hline & 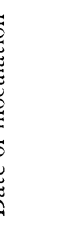 & $\begin{array}{l}\overrightarrow{\tilde{O}} \\
\overrightarrow{0} \\
\dot{a} \\
\vec{\Xi} \\
\vec{\Xi}\end{array}$ & 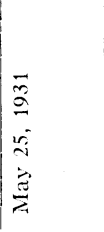 & 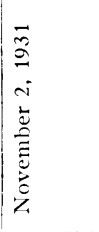 & 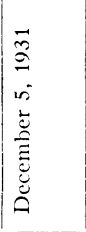 & 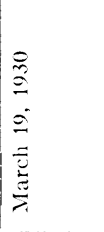 & 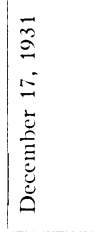 & 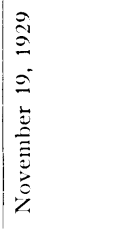 & 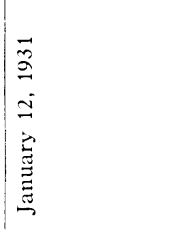 \\
\hline & & $\stackrel{\cong}{\Xi}$ & 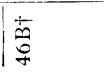 & $\hat{x}$ & $\infty$ & in & $1 \%$ & $\mid \vec{m}$ & 总 \\
\hline
\end{tabular}


O. H. Robertson, L. T. Coggeshall AND E. E. TERRELl 439

\begin{tabular}{|c|c|c|c|c|c|c|c|c|c|c|c|}
\hline \multicolumn{2}{|c|}{ 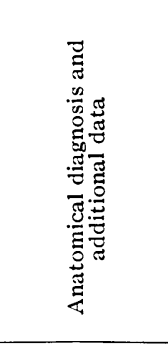 } & 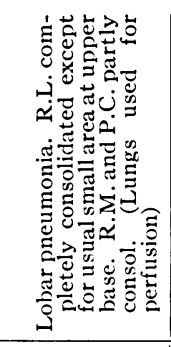 & 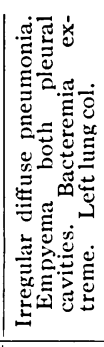 & 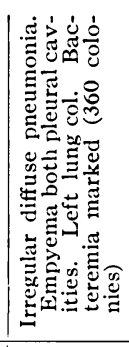 & 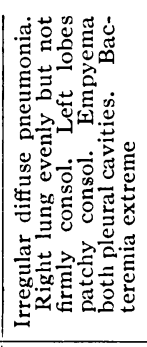 & 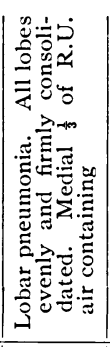 & 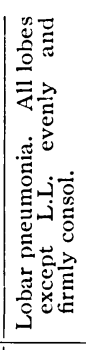 & 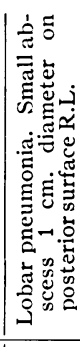 & 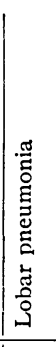 & 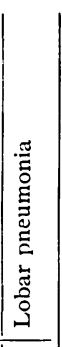 & \\
\hline \multirow{6}{*}{ 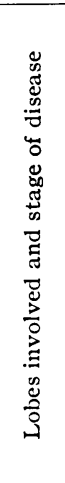 } & نْ & ن. & & & & & $\dot{\vec{j}}$ & & \begin{tabular}{|l|}
$\dot{\theta}$ \\
$\dddot{\Delta}$ \\
$\simeq$
\end{tabular} & 离 & \\
\hline & ن. & & i & $\dot{\bar{c}}$ & $\mid \overrightarrow{\check{x}}$ & & $\mid \begin{array}{l}= \\
\check{\sim}\end{array}$ & $\dot{\ddot{n}}$ & & $\approx$ & \\
\hline & $\dot{H}$ & & $\dot{0}$ & 13 & 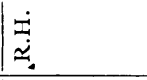 & & $\Leftrightarrow$ & & & & \\
\hline & $\dot{\sim}$ & & & $\Leftrightarrow$ & $\mid \begin{array}{l}\ddot{\not} \\
\check{\simeq}\end{array}$ & $\mid \underset{\ddot{\not}}{\ddot{\varkappa}}$ & $\mid \dot{\vec{j}}$ & \begin{tabular}{|l}
$\dot{\Xi}$ \\
$\simeq$ \\
$\simeq$
\end{tabular} & 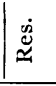 & & \\
\hline & 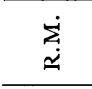 & $\dot{\vec{b}}$ & & 离 & $\stackrel{\vec{Z}}{\simeq}$ & $\dot{\overrightarrow{\ddot{n}}}$ & |ت & $\stackrel{\ddot{⿹}}{\check{\varkappa}}$ & \begin{tabular}{|l}
$\mathscr{0}$ \\
$\dddot{g}$ \\
$\simeq$
\end{tabular} & & \\
\hline & $\stackrel{\dot{\sim}}{\text { ¿ }}$ & $\underset{\dot{G}}{\dot{3}}$ & 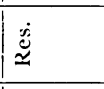 & $\begin{array}{l}\dot{\infty} \\
\stackrel{\mathscr{\varkappa}}{\sim}\end{array}$ & $\stackrel{\square}{\check{2}}$ & ن. & $\underset{5}{9}$ & $\stackrel{\dot{\Xi}}{\dddot{\Xi}}$ & & $\approx$ & \\
\hline \multirow{2}{*}{ 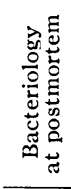 } & 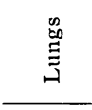 & & & $\dot{\Xi}$ & $\stackrel{\dot{\Xi}}{\dot{2}}$ & $\stackrel{\dot{I}}{2}$ & & & 0 & 0 & \\
\hline & 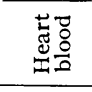 & & & $\dot{\equiv}$ & $\dot{=}$ & 0 & 10 & & & & \\
\hline \multirow{2}{*}{ 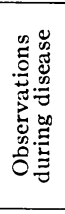 } & 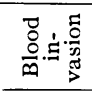 & 0 & + & + & + & 0 & + & 0 & 0 & + & \\
\hline & 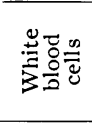 & 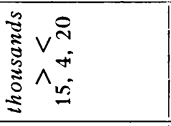 & $\begin{array}{l}v_{0}^{=} \\
\wedge_{0}^{-} \\
=\end{array}$ & $\begin{array}{r}\approx \\
V 0 \\
0\end{array}$ & $v_{0}^{\infty}$ & $v_{\stackrel{m}{m}}^{+}$ & & $\begin{array}{c}n \\
v \\
v \\
= \\
=\end{array}$ & $\mid \begin{array}{r}3 \\
v \\
0 \\
0 \\
0\end{array}$ & 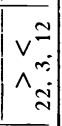 & $\stackrel{\dot{\Xi}}{\Xi}$ \\
\hline \multicolumn{2}{|c|}{ 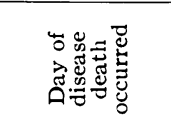 } & 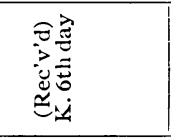 & 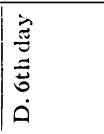 & 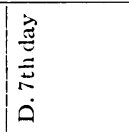 & 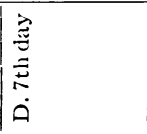 & 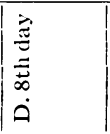 & 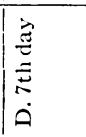 & 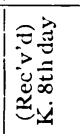 & 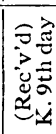 & 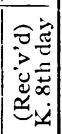 & $\begin{array}{l}E \\
\Xi \\
\vdots\end{array}$ \\
\hline \multicolumn{2}{|c|}{ 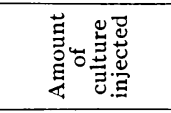 } & نั & $\simeq \overline{\overline{\dot{D}}}$ & 资 & $\overrightarrow{0}$ & 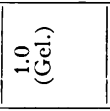 & 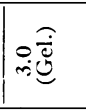 & $\overrightarrow{0}$ & $\ddot{3}$ & $\mid \begin{array}{l}20 \\
0 \\
0.00 \\
0\end{array}$ & \\
\hline \multicolumn{2}{|c|}{ 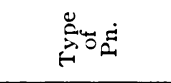 } & $\Xi$ & - & - & 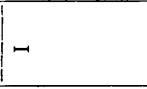 & - & $1-$ & - & $=$ & - & \\
\hline \multicolumn{2}{|c|}{ 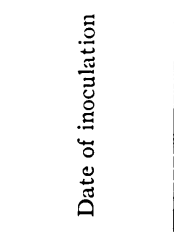 } & 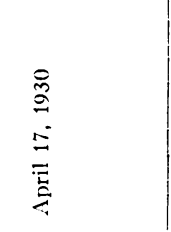 & 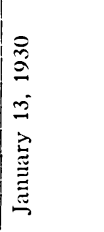 & 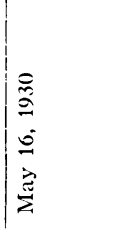 & $\begin{array}{l}0 \\
2 \\
\infty \\
2 \\
\overrightarrow{2}\end{array}$ & 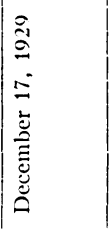 & 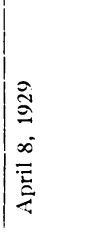 & 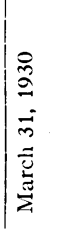 & 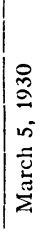 & 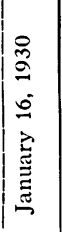 & 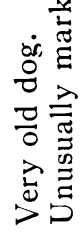 \\
\hline \multicolumn{2}{|c|}{ لّم } & 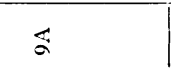 & I & $\mid \vec{z}$ & | & iq & 10 & F & in & | & \\
\hline
\end{tabular}




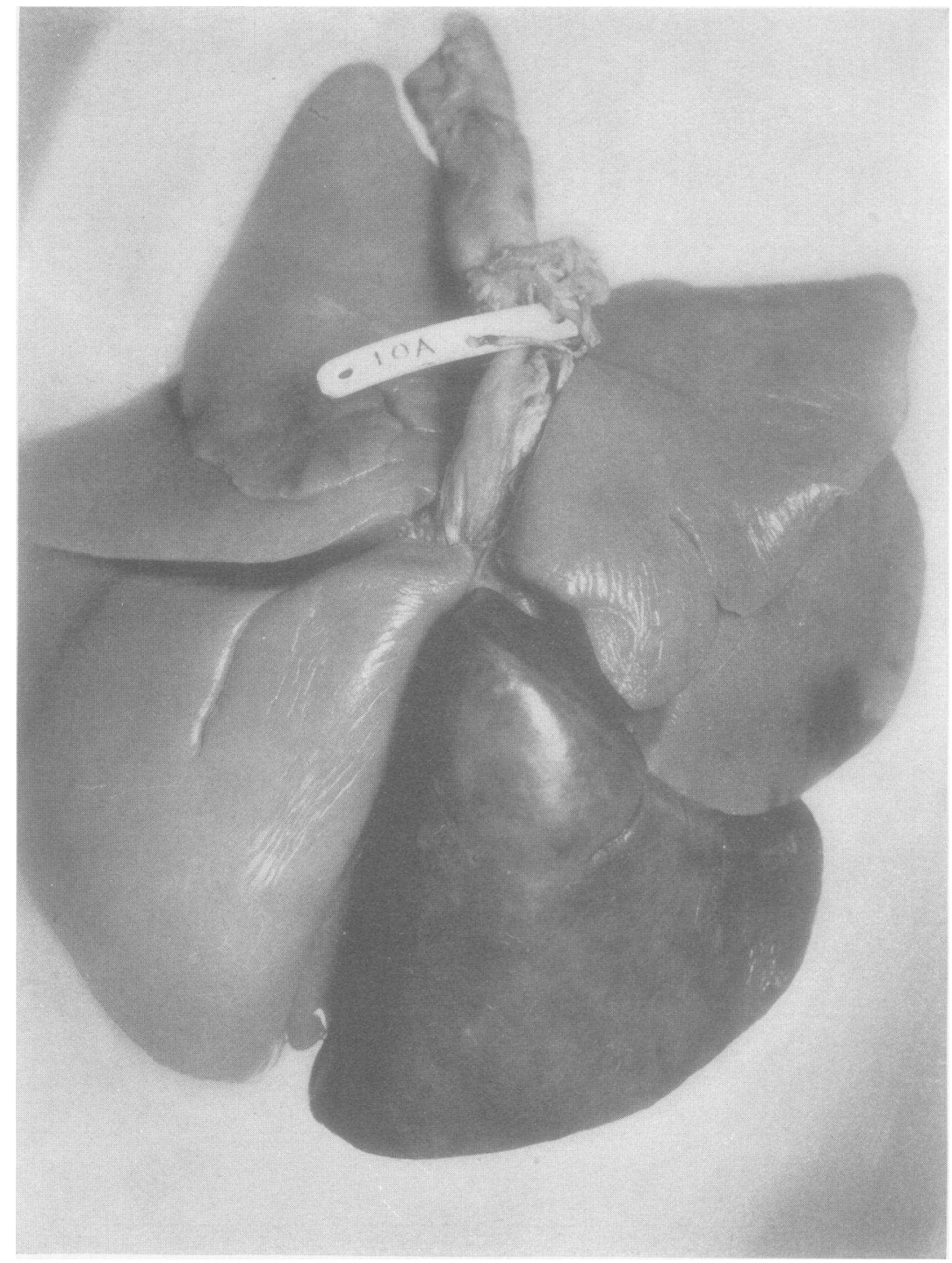

Fig. 1. Dog 10A. Lobar Pneumonia, Pneunococcus Type II: Killed at 48 Hours

Lungs perfused with normal salt solution to bring out contrast between normal and consolidated lobes. Posterior aspect of lungs showing lobar consolidation of right lower lobe and beginning consolidation of right middle lobe. Other lobes normal. 
O. H. ROBERTSON, L. T. COGGESHALL AND E. E. TERRELL

TABLE II

Summary of different types of lesion found at autopsy

\begin{tabular}{c|c|c|c}
\hline \hline \multirow{2}{*}{$\begin{array}{c}\text { Total number } \\
\text { autopsied }\end{array}$} & Number of dogs showing \\
\cline { 2 - 4 } & 20 & $\begin{array}{c}\text { Coalescent lobular } \\
\text { pneumonia }\end{array}$ & $\begin{array}{c}\text { Irregular diffuse } \\
\text { pneumonia }\end{array}$ \\
\hline 39 & 9 & 10 \\
\hline
\end{tabular}

* Includes dogs dying spontaneously and those killed from 48 hours to 8 days after the inception of the disease.

$\dagger$ Includes the occasional instance of non-coalescent lobular pneumonia.

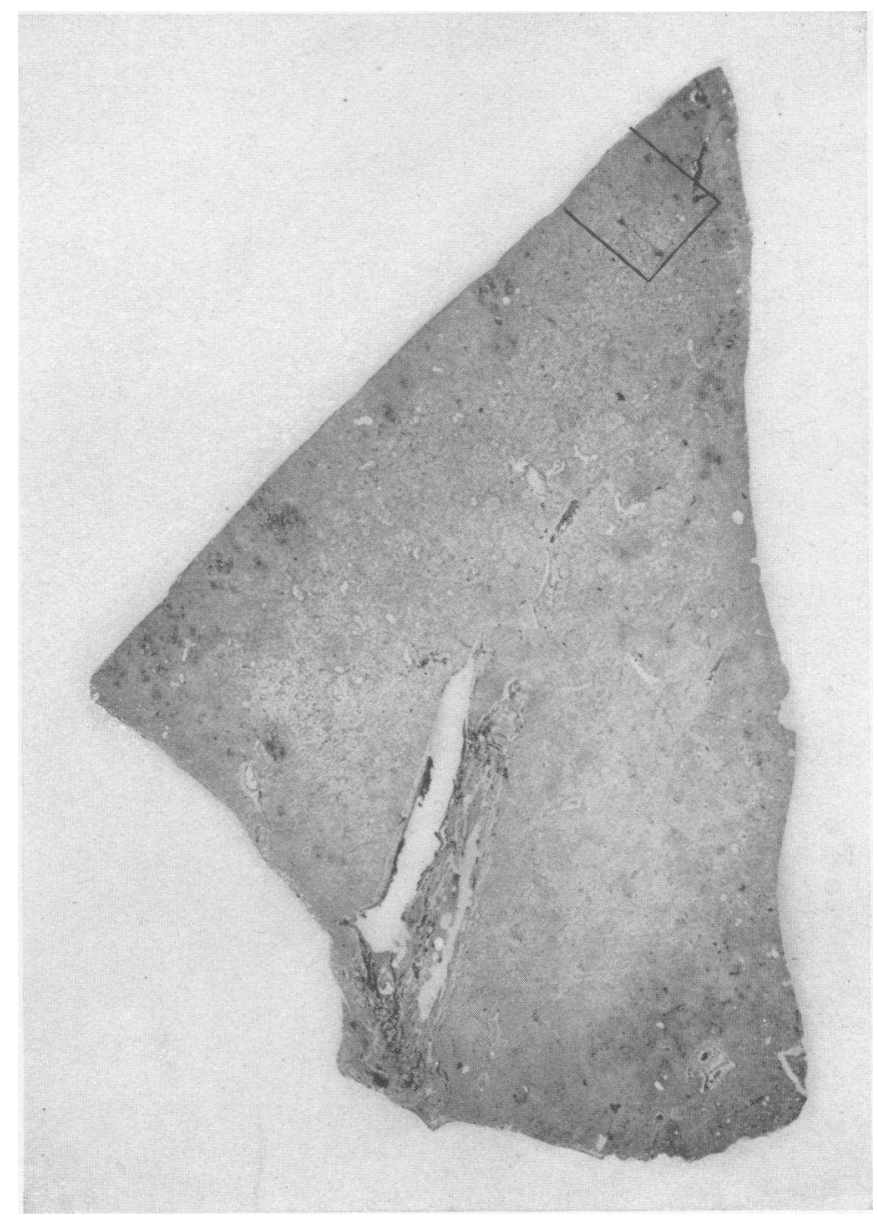

Fig. 2. Dog 14D. Lobar Pneumonia, Pneumococcus Type I; Killed at 48 Hours

Section comprises major portion of lobe and shows continuous and relatively even consolidation of whole lobe. $\times 3$. 


\section{Dogs showing localized lesions}

Macroscopic pathology. The lungs of animals dying from the characteristic experimental disease or sacrificed during its course presented a relatively uniform picture. Upon opening the thoracic cavity the con-

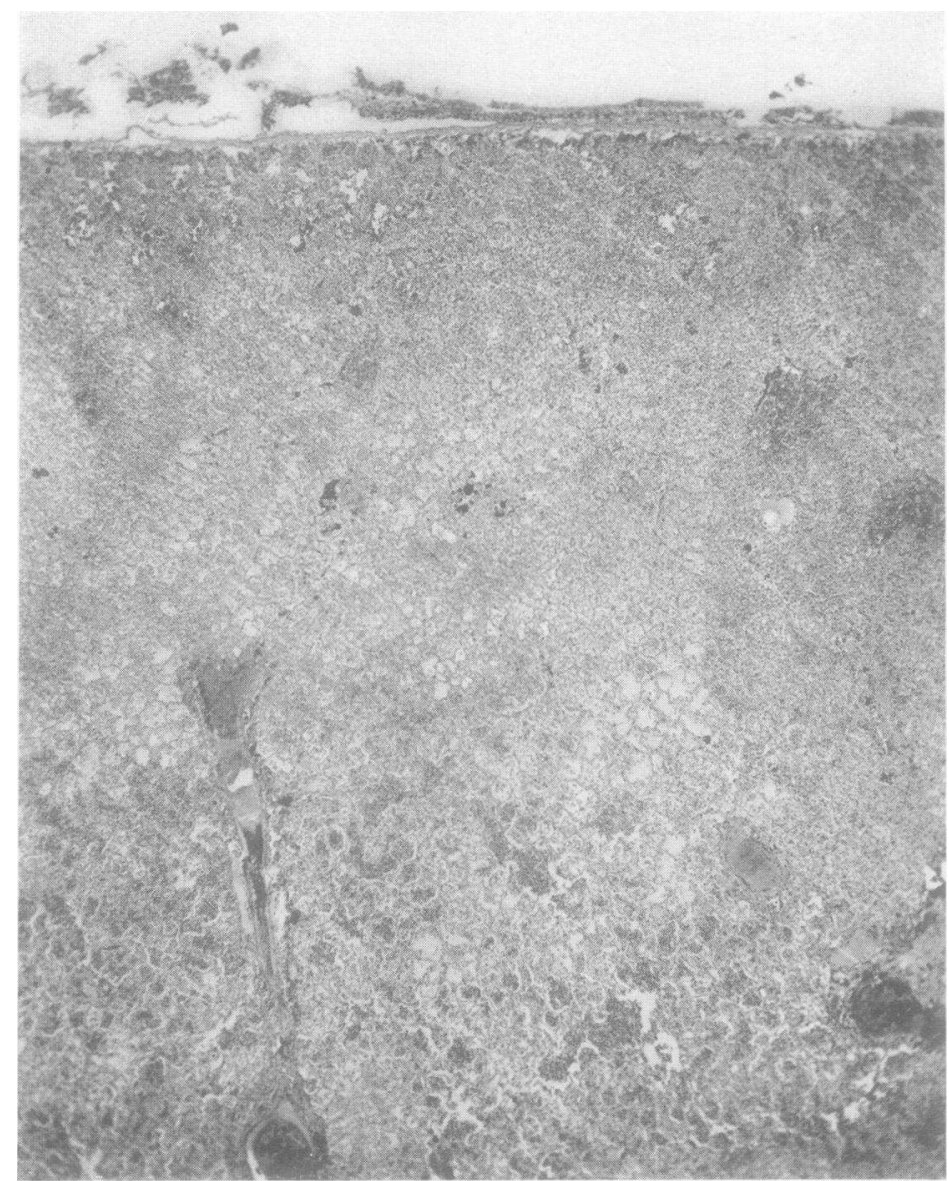

Fig. 3. Dog 14I), Lobar Pneumonia, Pneumococcus Type I

Higher magnification of marked area in Figure 2 showing character of infiltration and also exudate on surface of pleura. $\times 30$.

solidated lobes were seen to be of approximately the same size as the normally expanded lung - at times they were apparently larger (see previous paper, Fig. 17). Their color depended on the age of the process. In the early phase of engorgement the lobe was deep red. Later, corresponding to the stage of red hepatization, the color was a brownish red, which, after the fifth or sixth day of the disease, changed to a grayish brown or bluish gray. The degree of gray hepatization seen in human 
lobar pneumonia was never observed in the dog. The consolidated lobes were firm, airless and of liver-like consistency. The lesion involved the whole lobe or a continuous part of it (see Fig. 1, also Paper III, Fig. 15). In most instances, but not always, a thin layer of fibrin covered parts or the entire lung surface, not infrequently making adjacent affected lobes adherent. This fibrin could usually be peeled off. On cut surface the infiltration was seen to be even (Figs. 2 and 3). In the stage of engorgement it was moist, but in lobes showing red and early gray hepatization, dry and finely granular. In the later stages the bronchi frequently stood out above the surface. Fibrin plugs were often found in the smaller bronchi but somewhat less frequently than is seen in human lobar pneumonia. Dogs killed shortly after recovery showed the consolidated lobes in a state of resolution. They were somewhat smaller than normal, softer than in the previous stages, of ten partially air-containing and, on cut surface, sometimes showed much oozing of purulent material from the bronchi. The trachea and bronchi were either normal in appearance or only slightly injected, and contained varying amounts of mucopurulent, not infrequently blood-tinged exudate.

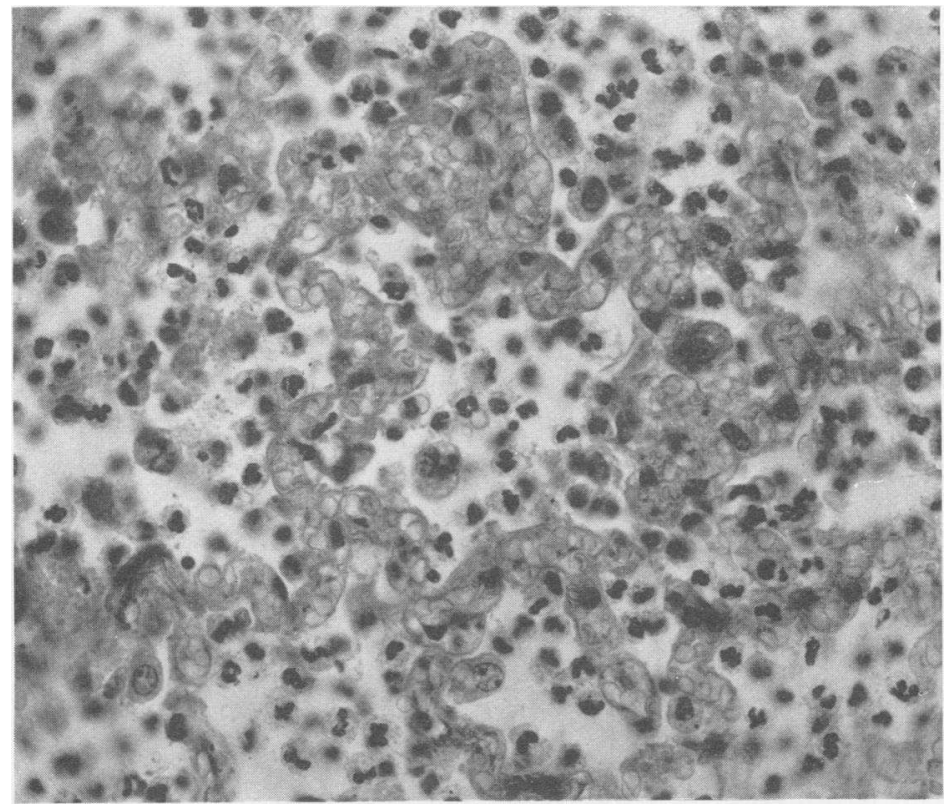

Fig. 4. Dog 40. Lobar Pneumonia, Pneumococcus Type I; Died on the 8Th DAY OF The Disease

Section from right upper lobe near spreading margin of lesion. Marked capillary dilatation present. Beginning cellular exudate, chiefly polymorphonuclear leukocytes. $\times 420$. 
As in patients dying with lobar pneumonia, the lungs of the dogs at autopsy showed various stages of the lesion in different lobes. The locus of beginning secondary involvement was quite inconstant. Sometimes this occurred in the center of the lobe, in others at the periphery or the hilum. Occasionally there appeared to be two or three points from which the spread had begun.

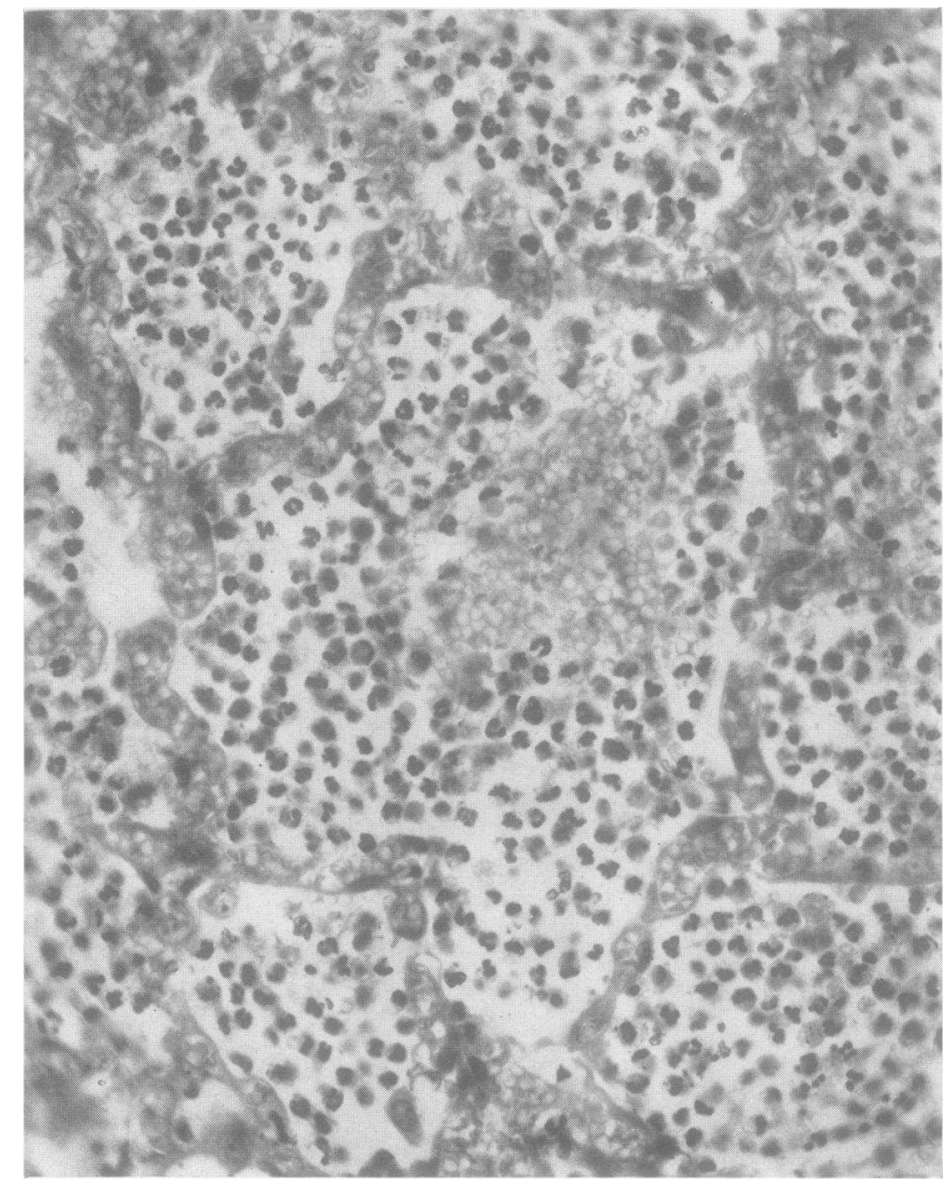

Fig. 5. Dog 40. Lobar Pneumonia, Pneumococcus Type I

Section from right upper lobe, showing stage of early red hepatization. A hemorrhage into the central alveolus is seen. $\times 380$.

Complications were not found in dogs dying from the disease course when the blood had remained sterile or had showed only a slight bacteremia. Empyema and other pyemic foci occurred only in those animals with an overwhelming blood invasion. The tracheobronchial lymph nodes at the roots of the involved lobes were enlarged and soft. 
Microscopic pathology. As the sequence of changes leading to complete consolidation of the pneumonic lesion will be dealt with in the section under pathogenesis, only the microscopic picture of the well developed lesion as found in animals after spontaneous death or in those killed after forty-eight hours will be described here.

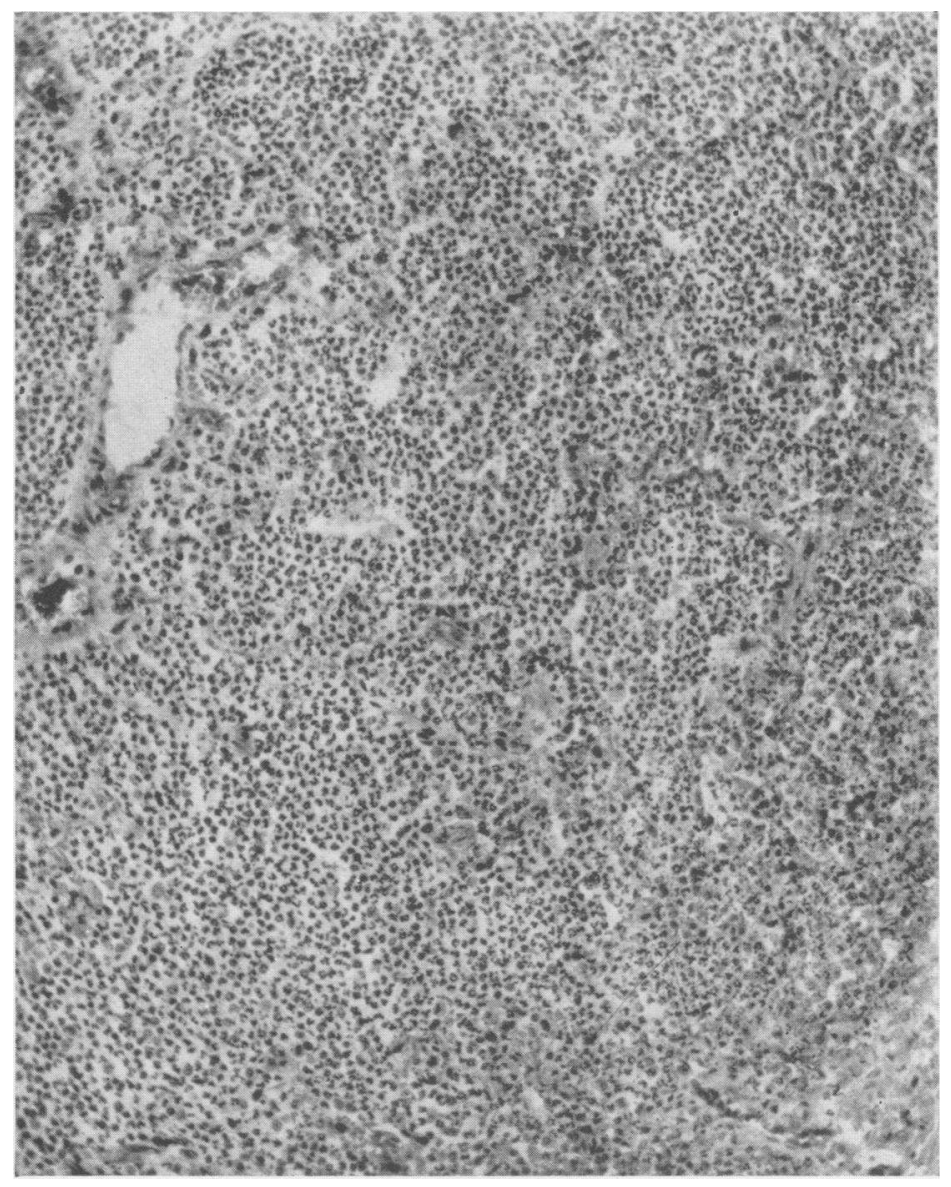

Fig. 6. Dog 21D. Lobar Pneumonia, Pneumococcus Type I; Killed on the 4th Day of the Disease

Section from right lower lobe showing the well advanced stage of red hepatization. Alveolar markings present but not conspicuous. $\times 165$.

Red hepatization. The stage of red hepatization, as seen at various periods, showed a progressively changing histological picture. The earliest phase, as observed near the margin of the spreading process (Fig. 4), showed a fairly even exudation of leukocytes into the alveoli, although the numbers of cells from alveolus to alveolus varied much. Some of the 
alveoli were quite small, contained only a few leukocytes and gave the appearance of partial collapse. Others were large and distended with cellular exudate. Here and there were clear spaces or lacunae with rounded margins which were interpreted as dilated air sacs. Edema

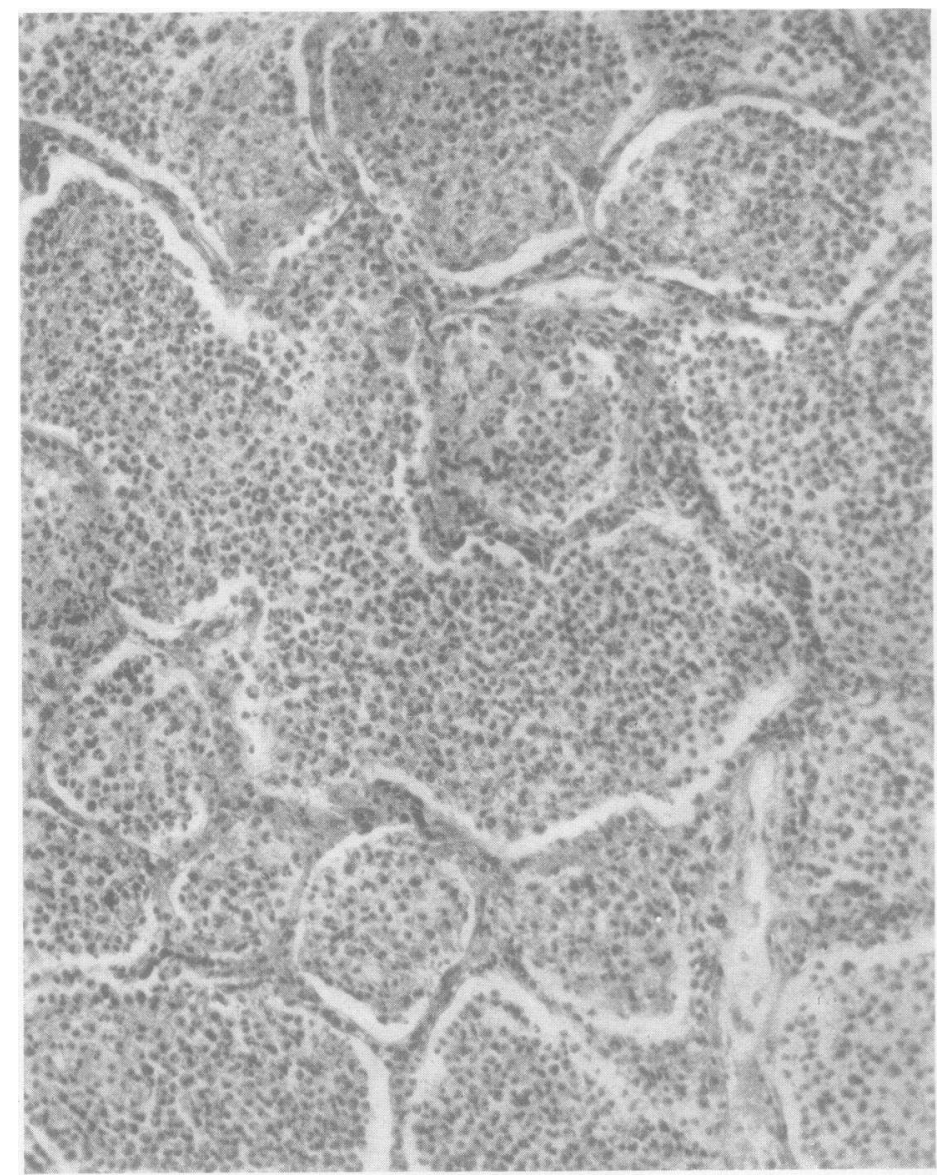

Fig. 7. Dog 49. Lobar Pneumonia, Pneumococel's Type II; DIED ON THE 3D DAY OF THE DISE.ISE

Right upper lobe showing red hepatization. The alveoli are markedly distended and their walls unusually promineit, giving the mosaic appearance of the typical picture of human lobar pneumonia. $\times 165$.

fluid was present to varying degrees in the alveoli. Occasionally an alveolus or a small group of alveoli was filled with fluid (Fig. 3, also see Paper III, Fig. 17). The architecture was generally well preserved. The cells of the exudate consisted chiefly of polynuclear leukocytes with here and there a lymphocyte and a large mononuclear cell with an eccentric nucleus which tended to be reticular. Some of these contained pig- 
ment. The prevalence of red blood cells varied much. In some areas very few were seen in the exudate; in others red cells were abundant. They were seen occasionally in groups as though there had been a hemorrhage into the alveolus, and indeed in places the capillary walls were seen to be ruptured (Fig. 5). Perhaps the most striking feature was the intense engorgement of the capillaries and the smaller blood vessels (Figs. 4 and 5). The large veins and arteries of ten showed slight to marked perivascular edema. The bronchi were filled with cellular exudate of the same character as that in the alveoli. The walls of some of the

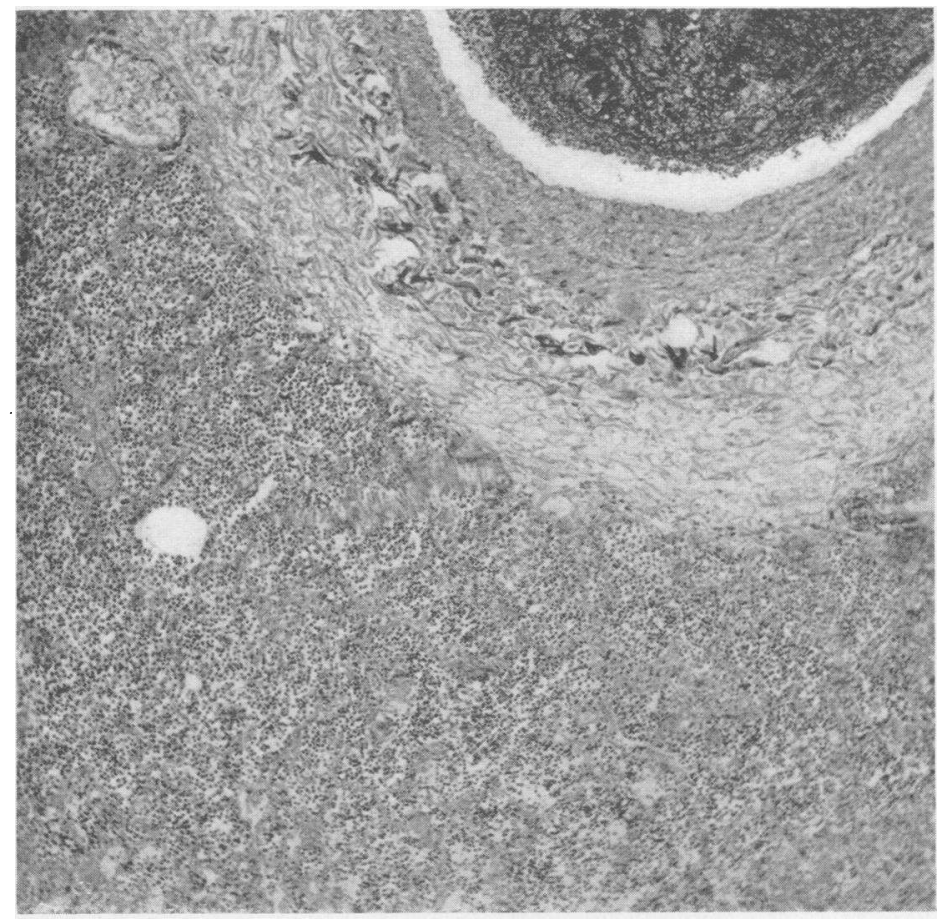

Fig. 8. Dog 40. Lobar Pneumonia, Pneumococcus Type I

Right upper lobe in stage of red hepatization showing marked edema of the wall of a large blood vessel. $\times 70$.

bronchi showed moderate amounts of edema. Fibrin began to appear early in the course of the lesion.

As the process developed, the chief changes were an increasingly uniform exudate of leukocytes into the alveoli. The partially closed alveoli observed in the earlier stage disappeared and the characteristic mosaic appearance of the human pneumonic lesion began to appear (Figs. 6 and 7). However, this was only occasionally as pronounced as in the human picture and infrequently evenly marked throughout a whole section. 
Figures 6 and 7 show the variations in architecture observed. The more usual appearance is that seen in Figure 6. There were still small scattered areas of edema but the exudate was predominantly uniform and cellular. Lacunae diminished in number. In some lesions they disappeared practically entirely and the picture was that of a uniform exudation (Figs. 3,6 and 7 ). In others, scattered lacunae were seen in different stages of red hepatization. The possible interpretation of these findings will be discussed later. The large blood vessels showed even more marked peri-

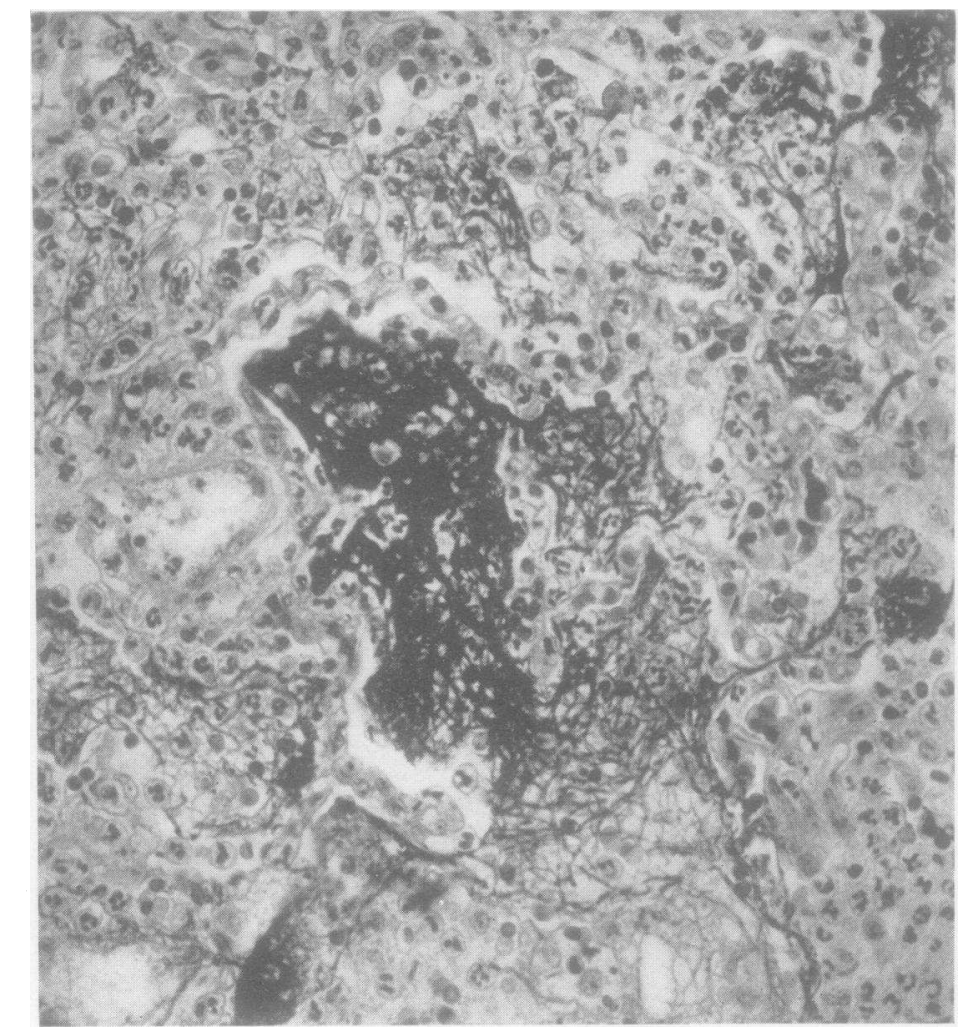

Fig. 9. Dog 56. Lobar Pneumonia, Pneumococcus Type II; Died on the 5Th Day of the Disease

Right lower lobe in the late stage of red hepatization showing characteristic deposition of fibrin. Note fibrin extending through alveolar pores. $\quad \times 380$.

vascular edema than was observed in the earlier lesions (Fig. 8). A few polynuclear leukocytes and monoculear cells were sometimes seen invading this zone and in certain instances frank perivascular hemorrhage was present.

The peribronchial tissues usually showed little further change except for invasion of the wall by polymorphonuclear leukocytes which appeared 
to pass through the mucosa into the lumen. The pleura showed thickening and infiltration with leukocytes and red blood cells and some fibrin, but the exudate was chiefly on the surface (Fig. 3). Here and there were small clumps of fibrin scattered irregularly through the lesion, and chiefly in the lumen of the alveoli. In some areas this was abundant (Fig. 9); in other large areas none was seen.

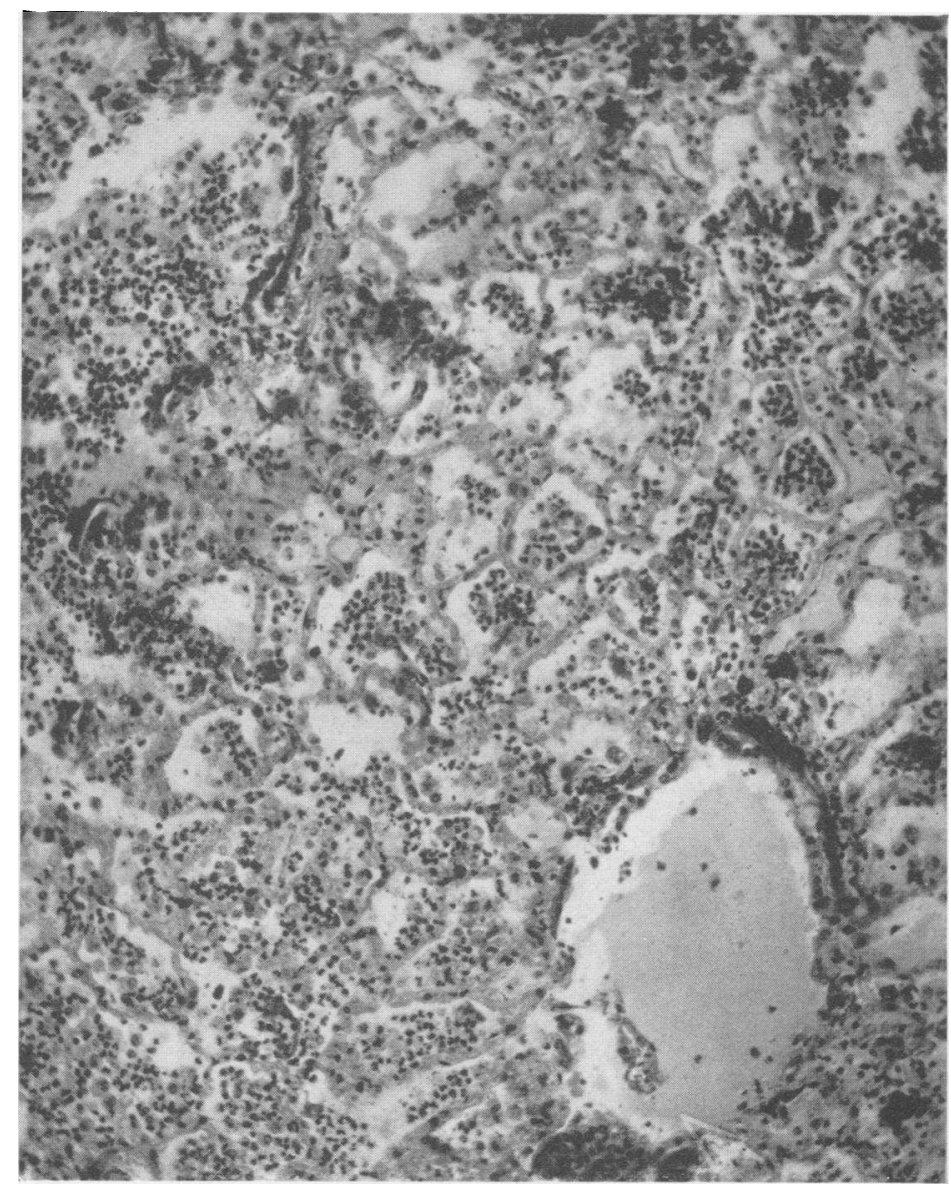

Fig. 10. Dog 56. Lobar Pneumonia, Pneumococcus Type II Right lower lobe showing an area of beginning resolution. $\times 140$.

The foregoing picture characterized the red stage as seen in most animals. The chief variations observed in the histological findings were in the relative amounts of red blood cells and edema in the exudate. In some instances considerable numbers of red cells persisted; in others they tended to diminish. Differences were observed in the same section from area to area. After the second or third day small areas of beginning 
resolution were frequently seen. This was accompanied by beginning re-aeration of the lobe as seen in the section of Dog 56, dying on the fifth day of the disease (Fig. 10).

Gray hepatization. This stage was not frequently seen. The most striking difference between red and gray hepatization was in the size of

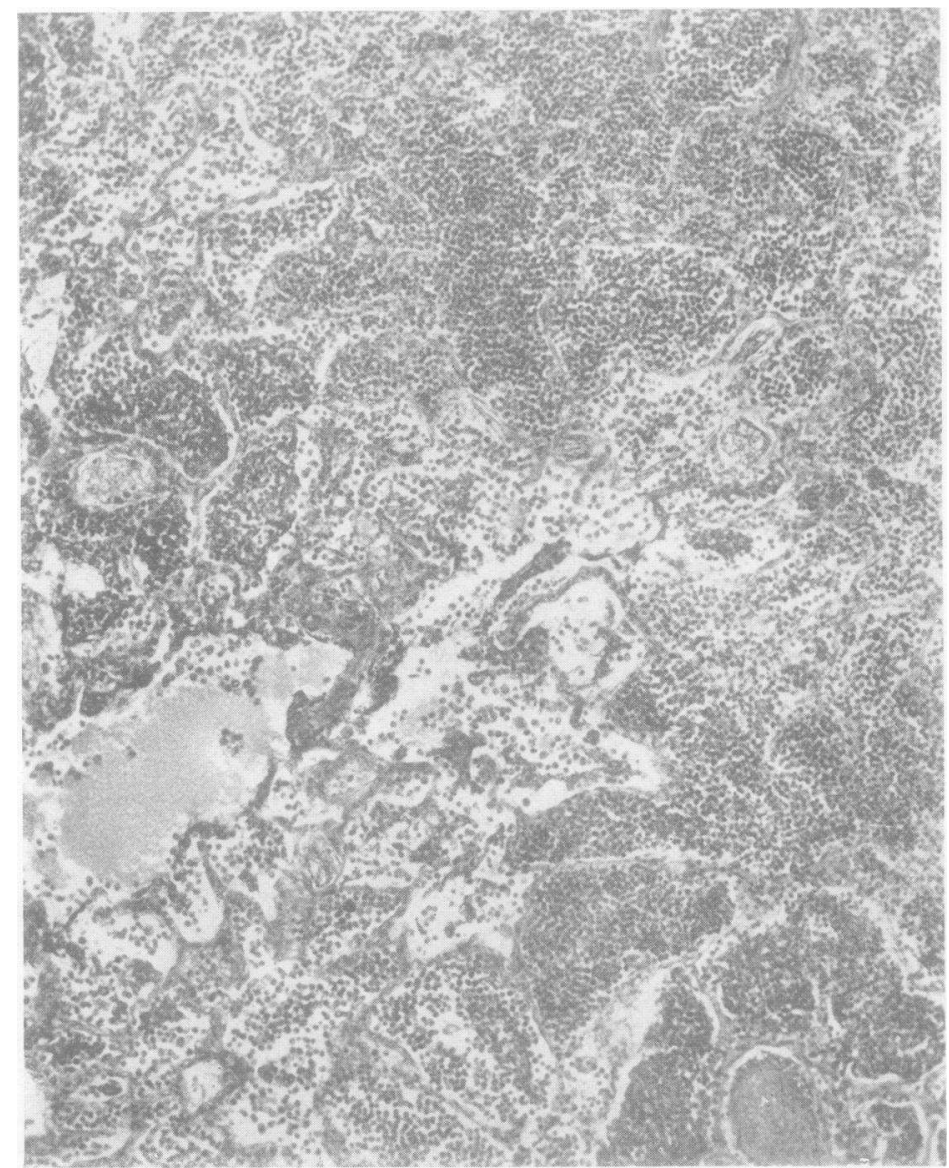

Fig. 11. Dog 16. Lobar Pneumonia, Pneumococces Type I; Died oN THE 7TH DAY OF THE Disease

Section from right lower lobe showing gray hepatization. Occasional areas of edema persisting. $\times 110$.

the alveoli. Whereas in the former, most of the alveoli were usually only moderately filled with cells, in the latter they were much distended (Fig. 12). The cells of the exudate were predominantly polymorphonuclear, although in places red blood cells still persisted. In some of the sections edematous alveoli or lobules were still seen scattered here and there (Fig. 11). The capillaries were usually well filled but were not as en- 
gorged as they were in red hepatization. In some sections the alveolar walls at this stage were quite thin. Fibrin thrombi of the smaller blood vessels and capillaries were seen only very occasionally.

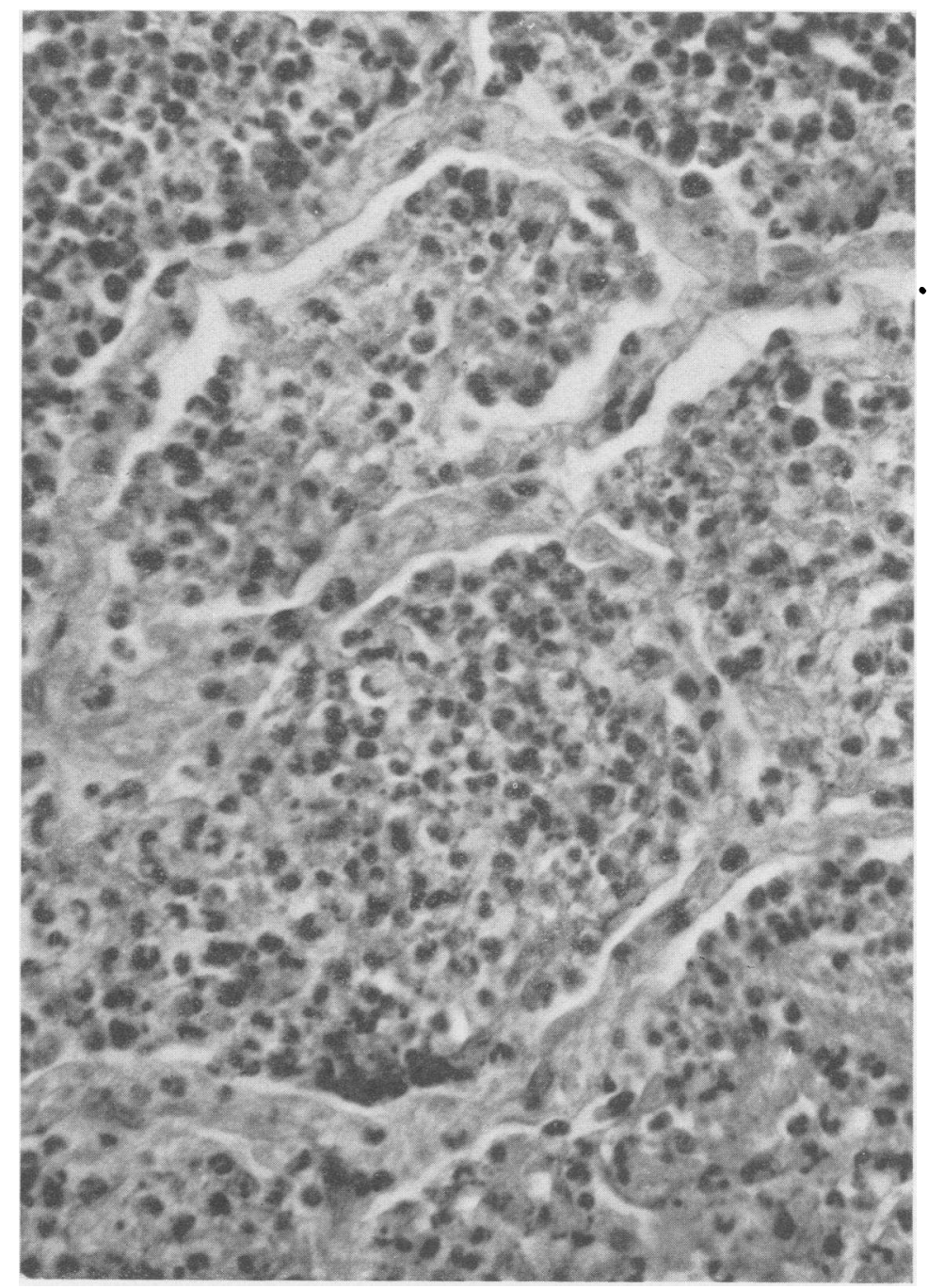

Fig. 12. Dog 16. Lobar Pneumonia, Fneumococcus Type I

Higher magnification of section shown in Figure 11. Alveoli much distended and alveolar walls prominent.

Resolution. The onset of resolution was characterized by what might be termed, for convenience, the "macrophage reaction." The first evidence of this change was a swelling of the cells of the alveolar wall in certain areas, either singly or in a row around the inner surface of the alveo- 
lus. This produced a very marked increase in the thickness of the alveolar walls (Fig. 13). Upon reaching a certain size these cells became detached from their fellows and wandered into the exudate as actively phagocytic cells (Fig. 14). The different stages of the resolving process were marked by the proportion of these macrophages in the exudate. Early in the process they were few in number but actively engulfing

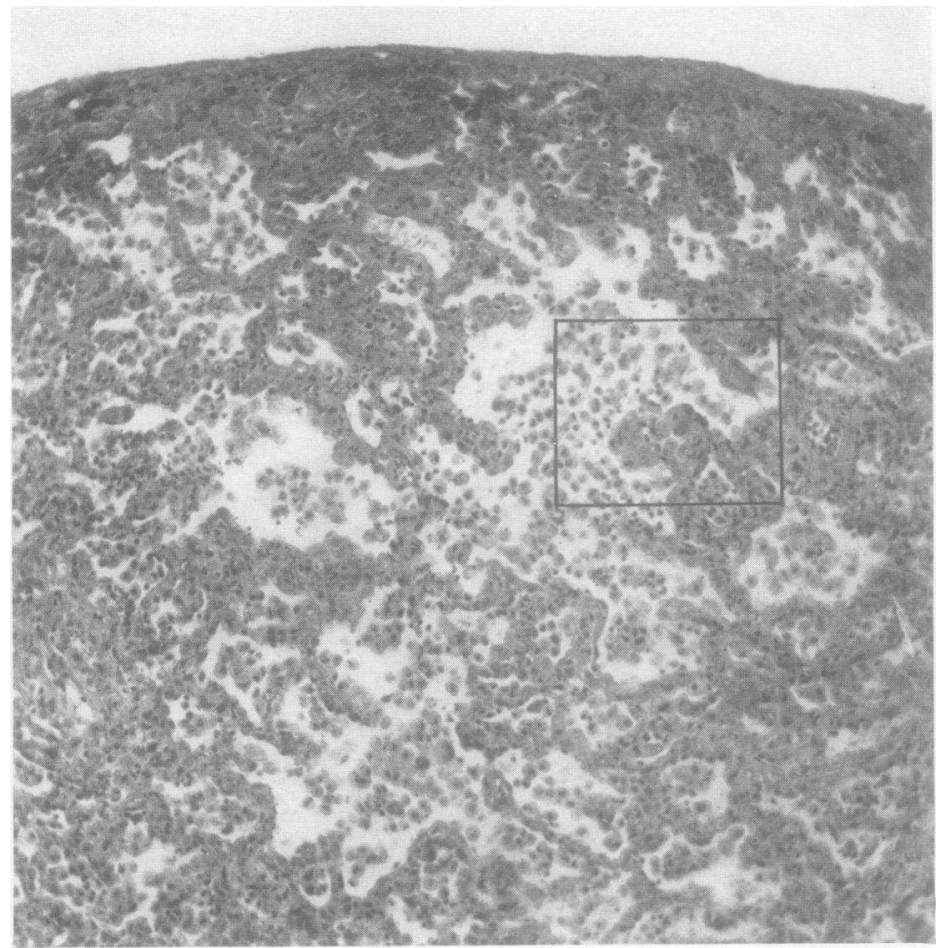

Fig. 13. Dog 53. Lobar Pneumonia, Pneumococcus Type II; Killed 2 Days after Recovers

Section from right middle lobe showing the picture of well advanced resolution. Alveolar septa markedly thickened. $\times 120$.

polynuclear leukocytes, red blood cells and pneumococci. Toward the end of the process they were practically the only cells seen in the exudate. Scattered among these newly detached cells were occasionally pigmentbearing macrophages. The fibrin during this time disintegrated and finally disappeared with the cellular exudate. Evidence of the breaking down of polynuclear leukocytes could also be seen. With the progress of clearing the alveoli diminished in size. Many became either partially or wholly collapsed. In most instances there appeared to be an increased engorgement of the capillaries at this stage, sometimes accompanied by exudation of red blood cells into the alveolar spaces. The bronchial 
exudate consisted of the same elements found in the alveoli but contained also, in some of the bronchi, numerous desquamated bronchial epithelial cells. Perivascular edema was still present, and to a considerably less extent, peribronchial edema, occurring only about the largest bronchi.

One of the most interesting features of this macrophage reaction was the tendency for it to occur at any time after the first twenty-four hours of the disease. Furthermore, just as is observed in lobar pneumonia in man,

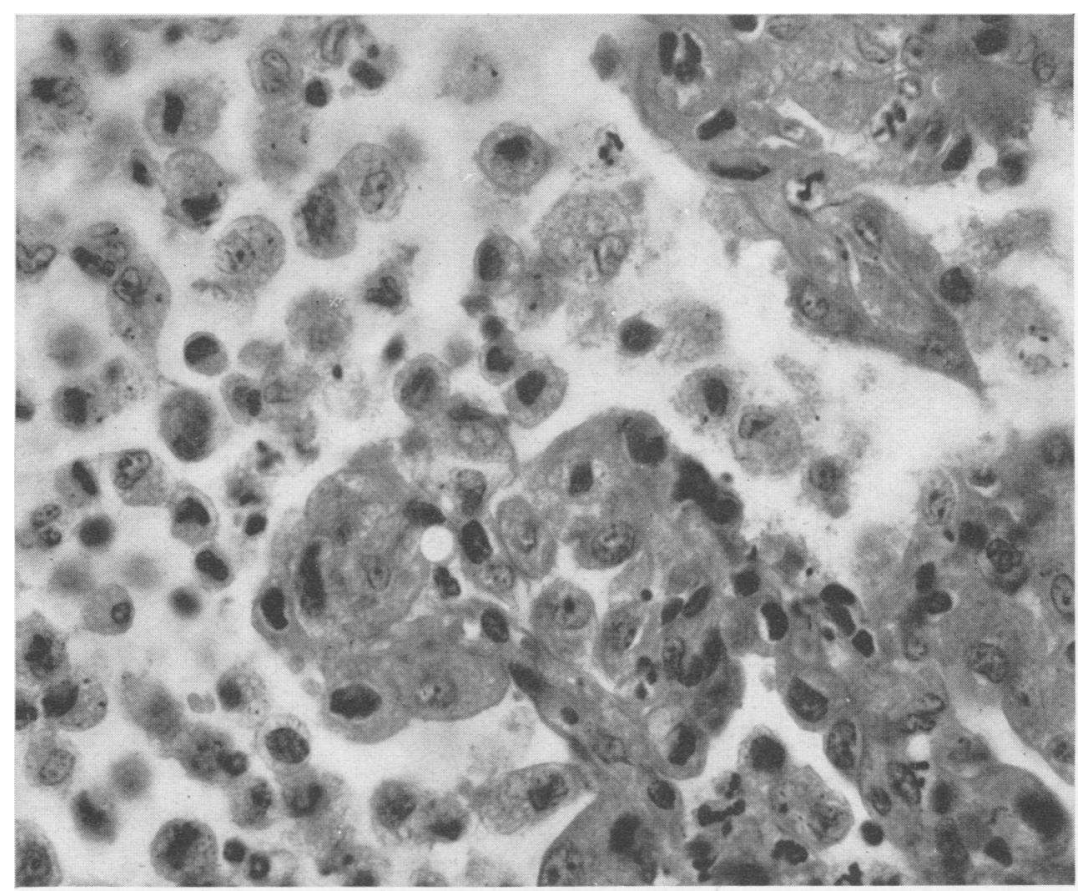

Fig. 14. Dog 53. Lobar Pneumonia, Pneumococcus Type II

High power view of area of Figure 13 enclosed in square. Cells of alveolar walls greatly enlarged and are becoming detached to form the large macrophages of the resolution exudate. $\times 625$.

certain areas of the lung were found to be in the stage of resolution at the time of death. Resolution was found regularly in the lungs of dogs killed at or just after recovery, but the areas of resolution were not always evenly distributed throughout the lung sections. Some areas showed an intense polymorphonuclear infiltration of the late red or gray type with the thin walled alveoli while others showed the typical and active macrophage reaction.

Organization. Small clumps of fibroblasts were seen in the parenchyma in those instances where the dogs lived as long as six days or were killed following recovery from a long illness. In one animal (Table 
I, Dog 4A) well marked organization was seen in the exudate within the pleura. Masses of fibroblasts were also observed in the peribronchial and perivascular tissues, particularly of the larger air tubes and blood vessels.

Distribution of pneumococci. The distribution and numbers of pneumococci in the lesions described varied with the age of the process. In the very early involvement, especially near the spreading edge of the lesion, pneumococci were abundant both in the alveoli, and to a lesser extent in the interstitial tissues where they were largely extracellular. In the areas of more intense cellular exudate they were fewer and more were intracellular. The older stages of red hepatization of ten showed numerous pneumococci but these were practically all intracellular. Many obviously were being digested as indicated by poor staining. Very few pneumococci were observed in the areas of resolution and these were entirely within the phagocyting cells.

\section{Fulminating type with early death}

Macroscopic pathology. The consolidated lobes of dogs dying within two to three days were found to be usually of the size of normally inflated lungs. In only two of nine animals dying early were the affected lobes definitely smaller than normal. As seen in Table I the process always involved two or more lobes. The lobes initially infected were deep red, usually entirely consolidated and airless. The secondarily involved lobes were more of ten only partly consolidated and frequently showed a patchy infiltration. The consolidated areas were usually, though not always, less firm than liver. The appearance of the cut surface varied. Sometimes it was uniformly deep red and moderately moist but in other cases it was mottled and oozed considerable quantities of serosanguineous fluid. $\mathrm{X}$-rays of the excised lungs, in some of these dogs, showed a fine mottling around the periphery of the lesion in contrast to the even shadow of the well localized process seen in Figure 17 of the preceding paper.

While blood invasion was the general rule, one of the eight animals showed a sterile heart's blood at autopsy. In three instances empyema occurred, and in one a beginning pleural effusion. In two dogs the lungs showed a mixed flora. No other complications were found. An early and marked drop in circulating leukocytes occurred as a rule in this type of the disease.

Microscopic pathology. The microscopic picture of this type of lesion differed from the well localized pneumonic process in several respects. First, the cellular exudate was less uniform in composition. Groups of alveoli containing cellular exudate alternated with areas of alveoli filled with edema or distended with air, or atelectatic. The distribution of the exudate suggested an infiltration of single or several primary lobules 
(Fig. 15). ${ }^{1}$ Secondly, the proportion of red blood cells in the exudate was much greater. Engorgement of the capillaries and smaller blood vessels was intense. Hemorrhages were not infrequent, sometimes of

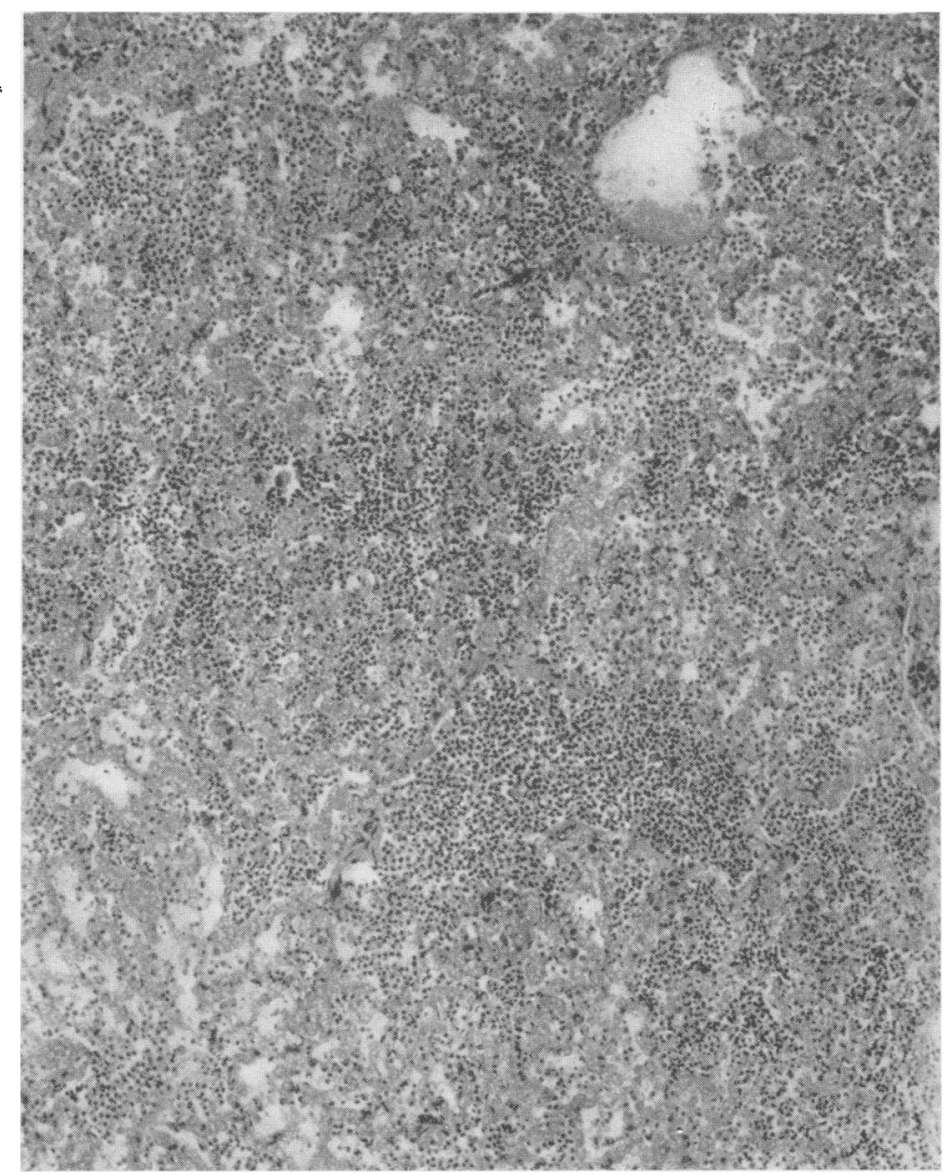

Fig. 15. Dog 28B. Coalescent lobular Pneumonia, Pneumococcus Type I; Died at 48 Hours

Section from right lower lobe showing the intra-alveolar cellular exudate to be chiefly lobular in distribution. $\times 107$.

considerable size, and apparently had occurred early in the development of the lesion. Thirdly, the architecture was less well preserved. Frequently in the large areas of edema or hemorrhage the alveolar walls were

${ }^{1}$ We have employed the anatomical classification of William Snow Miller (3), who defines a primary lobule or anatomical unit of the lung as consisting of the ductus alveolaris (terminal bronchiole) and its attached air sacs, each of which is subdivided into alveoli. The expanded portion of the ductus alveolaris leading into the air sacs is termed the atrium. 
partially or completely disrupted Lacunae were more numerous, and perivascular and peribronchial edema was more marked than in the well consolidated lung. The pleura was thickened and infiltrated. Pneumococci, both intra and extracellular, were seen in large numbers scattered throughout the lesion in the alveoli and interstitial tissues. The microscopical picture in dogs without bacteremia at death did not differ, in any noticeable way, from that of the other animals except that in these animals the pneumococci appeared to be for the most part intracellular.

This type of lesion has been designated as a coalescent lobular pneumonia (Table I). In only one animal, dying within three days (Dog 50), was the typical, intensely consolidated macroscopic and microscopic lesion of lobar pneumonia found throughout the lobes involved.

\section{Findings in dogs developing a generalized infection following an initial bocalized besion}

The lungs of dogs dying with an extreme degree of bacteremia presented a varied appearance. In some instances they were evenly, though not usually very firmly, consolidated. In some the lungs showed patchy consolidation and were partly air-containing. In others the lobes were definitely smaller than normal and appeared partially collapsed. In four cases, only a single lobe, the right lower, was affected. The other six animals showed two or more lobes involved. The color of the involved lobes was usually dark red brown and liver-like. Fibrin adhered to the surface. The cut surface had a mottled appearance, and oozed considerable serosanguineous fluid. When empyema was present the involved lobes were found collapsed to a greater or lesser extent.

Empyema was found in four of ten such dogs (Table I). In three instances the empyema was bilateral. In one case, Dog $8 \mathrm{~B}$, killed, before death the pleural cavities were clear but a pyopericardium was present. Three animals showed no focal complications.

Microscopic pathology. The microscopical examination of these lungs showed as varied a picture as was seen on gross inspection. The character of the lesion differed not only from lobe to lobe but in different parts of the same lobe. The histological picture was a combination of lobar consolidation, coalescent and noncoalescent lobular pneumonia, and, in addition, a suggestion of interstitial pneumonitis (Fig. 16). However, high power examination of the area depicted in Figure 16 showed that much of what appears to be interstitial exudation when viewed under low power, was actually a layer of intra-alveolar cells adherent to the alveolar wall. In places, well marked exudation of polymorphonuclear leukocytes and other cells was present in the alveolar septa. In some animals the appearance was more like that seen in the fulminating type of infection, and showed hemorrhages and other evidences of marked tissue injury, e.g., disruption of the architecture, an extreme degree of peribronchial and peri- 
vascular edema, and desquamation of the bronchial epithelium. In others the lobe or lobes involved were in a state of well advanced resolution. Collapsed alveoli were much in evidence, especially in those animals with empyema. Pneumococci were present in considerable numbers

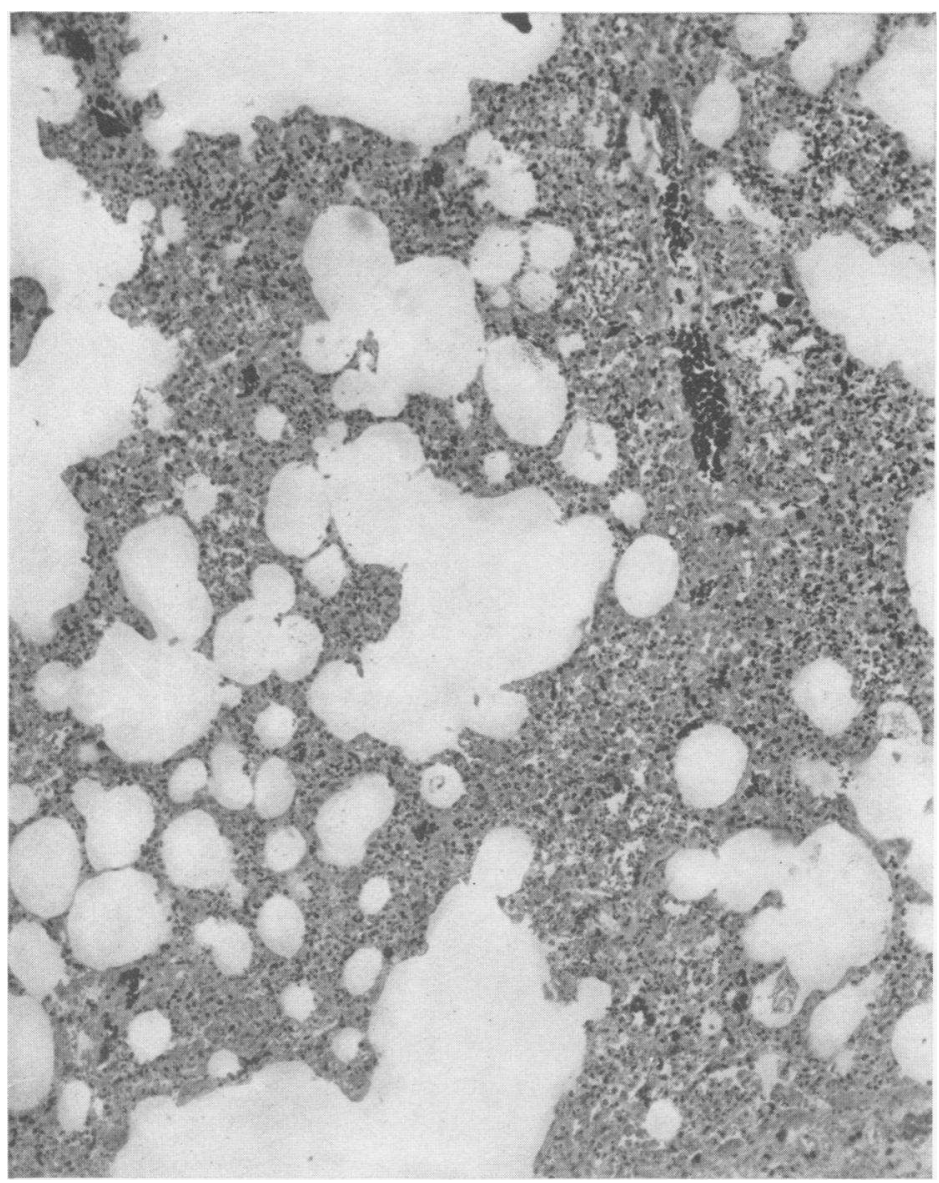

Fig. 16. Dog 4D. Irregular Diffuse Pneumonia, Pneumococcus Type I; Killed while Moribund on 4Th Day of Disease

Section from right lower lobe showing irregular distribution of cellular exudate which is sparse in amount. $\times 110$.

throughout the lesions, both intracellular and extracellular, except in the areas showing resolution where they were rare or absent, and apparently entirely within the phagocyting cells.

This type of lesion has been designated as an irregular diffuse pneumonia.

Examination of the brain and spinal cord, made in some of the dogs dying after a prolonged course, failed to reveal meningeal involvement. 


\section{PROTOCOLS}

The following protocols are illustrative of the three types of pathological lesions affecting the lungs, and described above.

Protocol 1. Dog 40, male, weight $20.8 \mathrm{kgm}$. December 17, 1929, the dog was injected intrabronchially with $1 \mathrm{cc}$. of pneumococcus Type I culture suspended in gelatin. The animal developed pneumonia, which remained localized in the right lower lobe until the fourth or fifth day of the disease when the $\mathrm{x}$-ray showed an extension of the process to the right middle lobe. A further extension to the right upper lobe was noted the day before death. There was an early leukopenia followed by leukocytosis. The blood remained sterile throughout the disease course, which terminated in death on the eighth day. Autopsy performed shortly after death.

Anatomical diagnosis. Lobar pneumonia of the right lower, middle and upper lobes. Right lower lobe, stage of gray hepatization and resolution; middle and upper lobes, red hepatization. Acute fibrous pleuritis.

Gross findings. The trachea was clamped before opening the chest. No fluid in either pleural cavity. The three lobes of the right lung filled the right pleural cavity, were completely and uniformly consolidated except for the medial portion of the upper lobe (see Fig. 17, Paper I for x-ray of excised lungs). The lobes of the left side and the post-cardiac lobe were normal. Scattered over the surface of the consolidated lobes were small patches of fibrin which in places produced adhesions between adjacent lobe surfaces and to the chest wall. The lower half of the right lower lobe was of a bluish gray color, the upper half reddish purple. The two other lobes of the right side were a reddish purple color. On section the lung was found to be firm, about the consistency of liver, of an even color, the same as that seen on the surface, and exuding a serosanguineous fluid. The bronchi of the lower and middle lobes were empty. Those of the upper lobe exuded a mucopurulent material.

The heart appeared normal. Aside from congestion no changes were observed in the viscera with the exception of the pancreas, on the surface of which were numerous small areas of fat necrosis.

Cultures. Heart's blood negative. Smears from the lung surface showed only pneumococci.

Microscopical examination. Right lower lobe. The pleura was only moderately thickened. This was due principally to marked engorgement of the vessels of the subpleural layer. However, in places there was edema with some cellular infiltration, polymorphonuclears, round cells and red blood cells. Very little fibrin was seen. The architecture of the lung was in general well preserved and the cellular exudate was evenly distributed throughout the section. However, not all the alveoli were equally filled with cells. Some were partly empty and this was related to the stages of the disease. The exudate consisted of polymorphonuclears, red cells and large mononuclears of the macrophage type. In many alveoli the macrophages predominated. They could be seen attached to the alveolar walls which were much thickened as the result of the swelling of the septal cells. Certain parts of the lobe showed the macrophage reaction to a marked degree. There was pronounced engorgement of the capillaries and small blood vessels. Perivascular and peribronchial edema was not marked. The epithelium of some of the bronchi showed signs of injury. The bronchial exudate consisted chiefly of polymorphonuclears but contained also macrophages and red cells. At the lower tip of the lobe in 
the area of initial consolidation where the process of resolution was most advanced, active organization was seen. Fibrin was seen fairly abundantly throughout the lesion in fine strands in the alveoli and the blood vessels, including the capillaries.

The microscopical picture of the middle and upper lobes differed from that of the lower lobe in several respects. (1) The intra-alveolar cellular exudate was distributed quite evenly throughout the sections (Figs. 5 and 8). This consisted of polymorphonuclears and red blood cells which were much more abundant than in the lower lobe. (2) Very few macrophages were seen. The septal cells were flattened as in normal lung tissue and hence the alveolar walls were thinner and more sharply defined. (3) Perivascular and peribronchial edema was more marked. Fibrin was less in amount, but the distribution was in general the same with the exception that fewer capillaries contained fibrin strands. A portion of the upper lobe at the junction of consolidated and normal tissue showed a very early stage of red hepatization (Fig. 4).

The numbers and distribution of pneumococci differed in the several lobes. In the lower lobe, showing beginning resolution, the pneumococci were few in number and apparently entirely intracellular. In the middle and upper lobes they were more numerous but also practically all intracellular. However, at the junction of consolidated and normal tissues in the upper lobe extracellular pneumococci were numerous.

Protocol 2. Dog 28B, male, weight $7.8 \mathrm{kgm}$. March 3, 1931, the dog was injected intrabronchially with 0.06 cc. of pneumococcus Type I culture suspended in starch. The animal showed marked and prolonged morphine depression. Temperature and pulse were still subnormal the morning of March 4. Developed pneumonia with pneumococcus septicemia and leukopenia. X-ray at the end of twenty-four hours showed a uniform consolidation of the right lower lobe. The remainder of the chest was clear. The dog died in less than forty-eight hours, probably only several hours before autopsy.

Anatomical diagnosis. Coalescent lobular pneumonia of the right lower, middle and upper lobes, as well as the left middle and post-cardiac lobes.

Gross findings. Before opening the chest the trachea was clamped. The pleural cavities contained no fluid. The right lower lobe was seen to be approximately the same size as the normal left lower lobe. It was firm and airless and uniformly consolidated except at the anterior lower margin where there was a small air containing area $1 \mathrm{~cm}$. in diameter. The right middle and upper lobes were irregularly consolidated. There was a small patch of consolidated tissue in the lower part of the left upper lobe, and another in the post-cardiac lobe. The remainder of the lung was normal in appearance. The cut section of the right lower lobe was of a deep red color showing a rather fine mottling. Much serosanguineous fluid oozed from the surface. The bronchi were not prominent. The consolidated areas in the other lobes had the same appearance. The other organs showed nothing of importance.

Cultures. Heart's blood and lungs gave an abundant growth of pneumococcus.

Microscopical examination. Right lower lobe. The pleura was moderately thickened showing an infiltration with polymorphonuclears and red blood cells. Occasional patches of fibrin were seen on its surface. The architecture was in general well preserved but in areas of hemorrhage and edema sometimes involving a number of alveoli, the alveolar walls were disrupted. Lacunae were present here and there. The exudate consisting principally of polymorphonuclear leukocytes and red blood cells was by no means evenly distributed. 
Under low power, groups of alveoli, frequẹtly seen to be surrounding an atrium, were completely or fairly well filled with cells, while other adjacent groups contained only a few exudated cells or edema fluid and many appeared to be partially collapsed (Fig. 15). Engorgement of the capillaries and smaller blood vessels was intense and frank hemorrhages were not infrequent. There was marked edema of the walls of the large blood vessels and bronchi. Some of the bronchi contained cellular exudate, others only edema fluid. The epithelium of the bronchi was in many places separated from the basement membrane and in some parts disrupted. Pneumococci, both intra and extracellular, were numerous throughout the inflammatory lesion. Phagocytosis was marked. Many polymorphonuclears contained a large number of pneumococci in all stages of digestion.

Protocol 3. Dog 4D, male, weight $13.6 \mathrm{kgm}$. October 22, 1931, the dog was injected intrabronchially with $0.25 \mathrm{cc}$. pneumococcus Type I culture suspended in starch. Developed pneumonia. On the second day of disease $\mathrm{x}$-ray showed involvement of the right lower and middle lobes as well as a beginning lesion in the right upper. On the third day the process occupied the entire right lung, and there was a marked displacement of the heart toward the right side. The blood remained sterile until the third day, when the blood culture showed 400 colonies per cc. On the fourth day the blood culture yielded 1,000 colonies per cc. There was a gradual drop in white count from 9,000 to 4,200 . The animal was very ill on the fourth day and was killed at this time and autopsy performed at once.

Anatomical diagnosis. Irregular diffuse pneumonia of the right lower, upper and post-cardiac lobes. Coalescent lobular pneumonia of right middle lobe.

Gross findings. The trachea was clamped. The pleural cavities contained no fluid. No fibrin was visible on lung surfaces. The three lobes of the right side and the post-cardiac lobe were consolidated and of approximately normal size. The consolidated lobes appeared to be evenly consolidated except for small areas of air containing tissue on the posterior surfaces of the upper and lower lobes. No crepitation except in these areas. The outer surface and cut section was of a dark chocolate color. A watery, frothy, chocolate brown colored fluid exuded from the cut lung. The left lobes showed no abnormal changes. Except for the usual congestion occurring in acute severe infection, the other organs appeared normal.

Cultures. Heart's blood and lung cultures gave an abundant growth of pneumococci.

Microscopical examination. Right lower lobe. The picture was a very irregular one. The cellular exudate was unevenly distributed. In some areas there was a fairly abundant polymorphonuclear intra-alveolar infiltration, for the most part lobular in character. Other areas showed edema, or only a few cells in the alveoli, many of which were partially collapsed. The alveolar markings did not stand out sharply. In places they were broken, particularly where hemorrhages were present. Lacunae were frequently met with. Resolution was beginning as shown by the well defined "macrophage reaction" occurring in many parts. Peribronchial and perivascular edema was slight. Fibrin was not abundant. The right middle lobe gave more the appearance of a coalescent lobular pneumonia. In places the distribution of the exudate approximated the character of the well consolidated lesion of lobar pneumonia. Fibrin was much more abundant than in the lower lobe, and peribronchial and perivascular edema was well marked. No evidence of resolution occurred. The right upper lobe revealed a younger lesion than the other lobes and in 
addition to the irregularity of distribution of exudate noted in the description of the lower lobe, showed what appears to be pronounced involvement of the interstitial tissues (Fig. 16). On close examination much of the apparent thickening of the alveolar walls was due to cells adherent to their surface. There was, however, a certain amount of infiltration both by cells and edema of the supporting frame work. A beginning "macrophage reaction" was observed here and there. Pneumococci were present throughout all sections, both intra and extracellular-fewest in the regions where resolution was occurring, and here they appeared to be almost entirely within the phagocyting cells.

\section{Bacteriology}

Cultures of the lungs and direct smears made from the cut surface showed only pneumococci in a surprisingly high percentage of the pneumonic lungs. This is of particular interest in view of the fact that a small gram negative bacillus can be cultured from the lungs of most normal dogs (4). In fact, in this series, cultures of normal lobes were found at times to yield this gram negative bacillus, while a pure culture of the pneumococcus was obtained from the consolidated lobe. Pneumococci were found regularly in the lungs of the consolidated lobes at autopsy, except in those instances where resolution was well established. Cultures of the throats and lungs of normal dogs failed uniformly to reveal the presence of pneumococci.

\section{DISCUSSION}

Interest in the pathology of experimental pneumococcus pneumonia in the dog centers chiefly in its relationship to the lesion of lobar pneumonia in man. The foregoing description of the findings in the dog's lung brings out certain striking similarities between the two pathological processes. In those animals exhibiting the typical clinical syndrome (1), in which the infection remained localized ${ }^{2}$ in the lungs throughout the course of the disease, the lesion was lobar in character, involving whole lobes or continuous parts of lobes. Consolidation was intense, producing a firm, airless, liver-like structure with a specific gravity greater than that of water. The histological features of the consolidated dog's lung resembled closely those found in human lobar pneumonia. The several stages of engorgement, red hepatization and gray hepatization were all observed in the evolution of the pulmonary lesion in the dog. Following resolution the structure of the lung regained its normal appearance except for an occasional small area in which organization had occurred.

There exist also well defined differences between the lesions of the experimental and the human disease. The consolidated dog's lung was usually not as firm to the touch as the lung of human lobar pneumonia, and the cut surface had a less granular appearance. The stage of gray

2 The term localization is employed to designate a pneumococcus inflammatory lesion from which there is at most only a slight detectable escape of pneumococci into the blood stream. 
hepatization was not as commonly observed in the experimental disease; death or recovery usually occurred before this stage was reached. It was only in animals living more than five days that gray hepatization was found. While a given area of a histological section of the diseased dog's lung might be indistinguishable from a similar stage of consolidation in the human tissue, examination of larger areas, or a whole lobe, would reveal certain features characterizing the process in the dog which are not typical of lobar pneumonia produced by pneumococcus Types I and II in the human being. In the first place, large sections of the dog's lung fail to show the uniformity of architectural delineation seen usually in the human being. Certain areas exhibit the typical mosaic appearance of the red or gray hepatized lung seen in man, but in others this is indistinct. The cellular exudation may be of the same character throughout but for some reason the alveolar walls are not sharply demarked as observed under low power. When examined under high power, however, they are seen to be intact but apparently thinner than those which are more easily detected. In the second place, the cellular exudate is rarely as evenly distributed in the dog as in the human being. In the well developed lesion in man alveoli filled with edema fluid are uncommon. On the contrary, they are found with considerable frequency in the dog throughout the whole evolution of the process. Thirdly, small lacunae (presumably dilated atria or air sacs) tend to persist in the consolidated dog's lung; in some animals being rare or absent, in others in fair numbers. Again, the degree of perivascular and peribronchial edema seen characteristically in the pulmonary lesion of the dog is more marked than is observed in man. Fibrin is much less abundant in the canine lesion, and is not frequently seen in the capillaries.

As compared with the human disease, the lesion in the dog tends to evolve more rapidly. This is particularly true of the process of recovery. While evidence of resolution of a portion of the consolidated area is sometimes seen fairly early in human cases, it is seen frequently in the experimental disease and, as pointed out previously, the "macrophage reaction," presumably indicative of local recovery, not uncommonly begins as early as twenty-four hours in, what appeared to be, a moderately severe infection. This presents interesting implications as to the nature of the recovery mechanism.

It seems not unlikely that these reactions which characterize the tissue of the dog's lung as varying from those of the typical human lesion may be due to differences in the anatomical structure of the lung of the two species. The dog's lung is a much more elastic and less fibrous organ than is the human lung. It has a very thin pleura and no clearly defined lobulation. When the negative pressure is removed it collapses to a far greater degree than does the lung of the human being. The alveolar septa are considerably thinner than those in the human being 
and the blood vessel walls appear to be more delicate. With such variations in structure, one would hardly expect to find, in these two species, identical reactions of the tissues to the invasion of a given pathogenic microorganism. However, the general features of the experimental disease, both clinical and pathological, resemble so closely the picture of lobar pneumonia in man as to permit the term lobar pneumonia of the dog for the characteristic experimental pneumococcus infection of lungs described above.

The pathology of experimental lobar pneumonia produced in the monkey by Blake and Cecil (5) and Shöbl and Sellards (6) and in rabbits by Permar (7) appears to differ in certain respects from that of the pneumococcus lesion observed by us in the dog. The marked and general primary inflammation of the supporting structures, as described by the above authors for the monkey and rabbit, is lacking in the pneumonic process in the dog. It is true that perivascular and peribronchial edema is prominent in the well developed lobar pneumonia in the dog, but inflammation of the smaller air passages and sacculi is an inconspicuous feature. Even in those types of the experimental canine disease where consolidation of the lung was irregular and obviously not a lobar pneumonia histologically, the picture presented is not typical of either an interstitial or a bronchopneumonia, although it would be difficult, in certain instances, to distinguish the process from a coalescent bronchopneumonia. It may be that the method of inoculation employed determines, at least, the initial character of the lesion. Intratracheal instillation of a pneumococcus culture, employed by the aforementioned authors, certainly produces an entirely different and much wider distribution of the infecting agent than occurs with starch suspension inserted into a terminal bronchus. The spread of the lesion from the hilum toward the periphery, as was observed in the monkey and rabbit, is in the reverse direction to the path of the initial lesion in our dogs. However, secondary lesions in other lobes of the dog's lung not infrequently began at the base and spread to the periphery, the pathology being in no detectable way different from that of the initial lesion.

The question may arise as to what relationship an experimental lesion, initiated at the periphery of the lung, may bear to lobar pneumonia in man which is believed to begin characteristically in the hilum region. While in the great majority of cases of lobar pneumonia the lesion appears to originate at or near the base of the lung, a certain percentage show initial foci of infection elsewhere. Recent serial x-ray studies by Graeser and $\mathrm{Wu}(9)$ in this clinic have demonstrated that the primary, or secondary lesions, of lobar pneumonia may begin in almost any region of the lung tissue, and spread from the original locus either toward the hilum or the periphery. As opposed to the current view of the locus of origin of the pneumonic lesion, Loeschcke (10) concludes, from his ex- 
tensive study of the pathology of lobar pneumonia in man, that the infection usually begins at or near the periphery of the lung.

An attempt was made to correlate certain observed changes during the life of the diseased animal with the course of the disease and the type of lesion found at autopsy. There appeared to be a fairly definite relationship between the degree of consolidation of the lung and the localization of the disease process. In those dogs showing a uniform consolidation with a well marked intra-alveolar cellular exudation, blood invasion was either absent, transient, or present to a moderate extent. On the contrary, irregular consolidation, giving the microscopical picture of lobular pneumonia or an irregular diffuse pneumonia, was, with rare exceptions, accompanied by bacteremia, of ten to an extreme degree. Comparative study of these lungs for the distribution of pneumococci showed that in the uniformly consolidated or lobar lesion, microorganisms were very largely intracellular, while in the irregularly consolidated ones pneumococci were present in greater numbers, and many lay free in the tissues and alveolar spaces.

The factors which are concerned in the production of the fulminating type of lesion appeared to be those which could be interpreted as having a depressant effect on the bodies' defense mechanism-namely, a large infecting dose or unusually prolonged action of morphine. The early and marked drop in the number of circulating leukocytes, observed in these cases (Table I), would explain, at least in part, the irregularity and incompleteness of the cellular infiltration. The hemorrhagic character of the lesion was further evidence of marked local toxic injury. More difficult to explain is the course of the disease in those animals in which the process began as a localized lesion, but failed to proceed to complete consolidation, and terminated with an extreme degree of bacteremia. In most of these dogs the dose was a lethal one; in others the infecting dose was one from which the majority of animals recovered. Usually there was an early leukopenia, but this did not always occur, and sometimes a leukocytosis was present. It may well be that an early diminution or loss of the pneumococcidal-promoting properties of the blood was the chief factor in determining the course of the disease in these animals. We know from former studies on this subject (8) that the pneumococcidal promoting properties of the blood serum disappear before bacteremia takes place.

\section{SUMMARY}

A study of the lungs of dogs dying of experimental pneumococcus pneumonia, or sacrificed during the course of the disease, revealed several distinct types of lesion which were associated with the different manifestations of the infection observed during life. The dogs in which the pathological process remained localized to the lungs throughout the course of the disease, and these constituted the majority of the animals, showed 
pathological changes in the lungs, similar, in their general features, to those of lobar pneumonia in the human being. The lesion was lobar in character, involving whole or continuous parts of lobes, and had a firm, airless, liver-like consistency. Consolidation of as many as four or five of the six or seven lobes of the dog's lung not infrequently occurred. The histological characteristics of the lesion in the dog resembled closely the picture of lobar pneumonia in man. There was the same type of vascular and cellular response; the stages of engorgement, red hepatization and gray hepatization were all observed and resolution occurred at the end of the disease leaving the lung parenchyma intact. The experimental lesion differed in certain respects from the process in man. Evolution of the lesion in the dog tended to progress more rapidly, with the result that resolution usually occurred before the stage of gray hepatization was reached. Microscopical examination of large areas of the consolidated lung in the dog failed to reveal the same uniformity of architectural delineation, and evenness of cellular exudation as is seen typically in lobar pneumonia in man. Other minor differences, chiefly in the degree of inflammatory reaction, were also observed. However, it was felt that the similarities between the experimental lesions in the dog and the natural process in man were sufficiently striking to permit the use of the term lobar pneumonia for the characteristic localized pneumococcus infection of lungs described in dogs.

Those animals in which the infection became generalized, either early or late in the course of the experimental disease, exhibited an entirely different pathological process. In the fulminating type of infection, characterized by marked bacteremia and early death, the picture was that of a coalescent lobular pneumonia accompanied by an unusual degree of injury to the tissues. When the generalized infection progressed more slowly and death occurred in four to seven days, with an extreme degree of bacteremia and usually pyemic complications, the lungs were found to be irregularly consolidated, sometimes partly collapsed; and the microscopical picture was a combination of lobar, coalescent and noncoalescent lobular pneumonia, with a suggestion of interstitial pneumonia. This type of lesion was designated as an irregular diffuse pneumonia.

\section{BIBLIOGRAPHY}

(1) Terrell, E. E., Robertson, O. H., and Coggeshall, L. T., J. Clin. Invest., 1933, xii, 393. Experimental Pneumococcus Lobar Pneumonia in the Dog. I. Method of Production and Course of the Disease.

(2) Wallace, H. M., Science, 1931, lxxiv, 369. A Stain for Fibrin, Gram Positive Bacteria and Basal Bodies in Tissues.

(3) Miller, William Snow, Am. Rev. of Tuberc., 1919-1920, iii, 65. Studies on Tuberculous Infection. II. A Description of Plastic Models (Reconstructions) of a Conglomerate Tubercle and the Surrounding Structures in a Human Lung. 
(4) Livingstone, H., and Adams, W. E., J. Infect. Dis., 1931, xlviii, 282. Bacterial Flora of the Lower Respiratory System of Normal Dogs.

(5) Blake, F. G., and Cecil, R. L., J. Exper. Med., 1920, xxxi, 445. Studies on Experimental Pneumonia. II. Pathology and Pathogenesis of Pneumococcus Lobar Pneumonia in Monkeys.

(6) Schöbl, O., and Sellards, A. W., Philippine J. Sc., 1926, xxxi, 1. Experimental Pneumonia in Monkeys.

(7) Permar, H. H., J. Ned. Research, 1923, xliv, 1. The Pathogenesis of Experimental Pneumonia in the Rabbit.

(8) Terrell, E. F., J. Exper. Med., 1930, li, 425. Changes in Humoral Immunity Occurring during the Early Stages of Experimental Pneumococcus Infection.

(9) Graeser, J. B., and $\mathrm{Wu}$, Ching. Physical Signs and X-ray Findings in Lobar Pneumonia. To be published.

(10) Loeschcke, H., Beit. z. path. Anat. u. z. allg. Path., 1931, lxxxvi, 201. Untersuchungen über die kruppöse Pneumonie. 